\title{
All-sky search for short gravitational-wave bursts in the third Advanced LIGO and Advanced Virgo run
}

\author{
R. Abbott et al. ${ }^{*}$ \\ (The LIGO Scientific Collaboration, the Virgo Collaboration, and the KAGRA Collaboration)
}

(Received 8 July 2021; accepted 12 October 2021; published 23 December 2021)

\begin{abstract}
This paper presents the results of a search for generic short-duration gravitational-wave transients in data from the third observing run of Advanced LIGO and Advanced Virgo. Transients with durations of milliseconds to a few seconds in the $24-4096 \mathrm{~Hz}$ frequency band are targeted by the search, with no assumptions made regarding the incoming signal direction, polarization, or morphology. Gravitational waves from compact binary coalescences that have been identified by other targeted analyses are detected, but no statistically significant evidence for other gravitational wave bursts is found. Sensitivities to a variety of signals are presented. These include updated upper limits on the source rate density as a function of the characteristic frequency of the signal, which are roughly an order of magnitude better than previous upper limits. This search is sensitive to sources radiating as little as $\sim 10^{-10} M_{\odot} c^{2}$ in gravitational waves at $\sim 70 \mathrm{~Hz}$ from a distance of $10 \mathrm{kpc}$, with $50 \%$ detection efficiency at a false alarm rate of one per century. The sensitivity of this search to two plausible astrophysical sources is estimated: neutron star $f$ modes, which may be excited by pulsar glitches, as well as selected core-collapse supernova models.
\end{abstract}

DOI: 10.1103/PhysRevD.104.122004

\section{INTRODUCTION}

The third observing run (O3) of the Advanced LIGO [1] and Advanced Virgo [2] detectors started on April 1, 2019, and ended on March 27, 2020. During O3, tens of gravitational waves (GWs) from compact binary coalescence (CBC) were detected [3-6]. In addition to CBCs, there are several plausible sources of short-duration GW transients (GW bursts) that have not yet been observed, such as corecollapse supernovae (CCSNe), neutron star excitations, nonlinear memory effects, or cosmic string cusps and kinks [7-11]. Additional source populations could exist that are yet to be predicted. For these reasons, GW burst searches capable of detecting a wide range of signal waveforms provide a unique opportunity for new discoveries.

All-sky searches look for signals arriving at any time from any sky direction. GW searches may use signal models (targeted search) or remain agnostic about the signal morphology (generic search). Targeted analyses include searches for CBCs [3-5,12] and cosmic strings [11]. Generic all-sky searches look for short-duration GW transients, up to a few seconds duration [13], and for longer GW transients, up to $\sim 10^{3} \mathrm{~s}$ duration [14].

This paper presents results of the generic all-sky search that is sensitive to the widest range of morphologies of short duration GW bursts during O3. The generic all-sky search is also sensitive to some CBC events [13], but these are not the primary targets of this analysis, and details of $\mathrm{CBC}$

*Full author list given at the end of the article. detections during $\mathrm{O} 3$ are given elsewhere $[3,4]$. Once the CBC candidates are excised, this search produces a null result.

This null result is interpreted in terms of sensitivities to a wide variety of generic morphologies, similarly to what was done in previous observing runs, $\mathrm{O} 1$ [15] and $\mathrm{O} 2$ [13]. The current analysis improves on previous upper limits due to improvements in detector sensitivity and a longer observation run. In addition, this paper includes the interpretation of results in terms of two expected astrophysical sources: $\mathrm{CCSNe}$ and neutron star $f$ modes. Since no tuning of the generic all-sky search is performed, these results should be considered conservative. The sensitivity of the search to five CCSNe waveform models is presented, both versus distance and for a Galactic distribution of sources. GW emission from CCSNe is expected in the frequency band below $1 \mathrm{kHz}$. Neutron star $f$ modes may be excited by pulsar glitches and are expected to emit GWs in the frequency range $2-3 \mathrm{kHz}$. The search sensitivity is tested for two equations of state and masses in the range $1-2 M_{\odot}$.

The analyses described here use the final LIGO-Virgo calibration [16-19] and data quality [20] information and their results supersede those from searches for GW bursts that were deployed in low latency during O3. The latter provided prompt public alerts for follow-up observations by other telescopes [21], analyzing near real-time data streams with preliminary calibration and data quality information.

The rest of this paper is organized as follows: Sec. II reviews the dataset used for these analyses. Section III describes the two search algorithms deployed and their 
results (III A), and discusses the loudest candidate events remaining after excluding the recognized $\mathrm{CBC}$ candidates (III B). Section IV discusses the sensitivity of this all-sky search and sets new rate-density limits for transient GW events other than $\mathrm{CBC}$, as well as the sensitivity to CCSN models and to neutron star $f$ modes. Finally, Sec. V summarizes the results and implications from this minimally modeled search for GW transients.

\section{O3: THE THIRD ADVANCED-DETECTOR OBSERVING RUN}

\section{A. Dataset}

The O3 dataset extends from April 1, 2019 to March 27, 2020. A commissioning break between October 1, 2019 and November 1, 2019 separates the first six-month epoch (O3a) of the observing run from the second epoch (O3b). Figure 1 shows typical spectral sensitivities of the detectors. The Hanford-Livingston (HL) network is analyzed during times where these two detectors operated in coincidence. In addition, results for the Hanford-Virgo network (HV) and the Livingston-Virgo network (LV) are presented for times when data from either of the LIGO detectors is not available. See Sec. III for an explanation of why the two detector network is preferred over the three detector Hanford-Livingston-Virgo (HLV) network for this search.

During the six months of O3a, 130.2 days of data were collected at Hanford, 138.5 days of data were collected at Livingston, and 139.5 days of data were collected at Virgo. The amount of data actually analyzed is reduced by requiring coincidence between two detectors, removing poor periods of data quality as described in Sec. II B, and requiring at least 200 seconds of continuous observation-quality data. This results in the following total amounts of analyzed data:

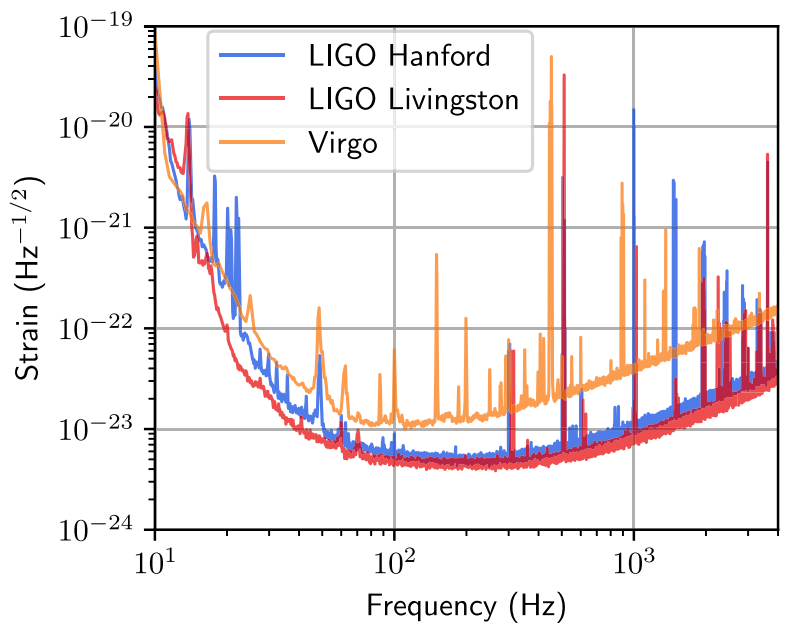

FIG. 1. Representative amplitude spectral density of the three detectors' strain sensitivity (LIGO Livingston 5 September 2019 20:53 UTC, LIGO Hanford 29 April 2019 11:47 UTC, Virgo 10 April 2019 00:34 UTC).
104.9 days for the HL network, 14.8 days for the HV, and 25.6 days for the LV network.

During O3b, data were collected for 115.7 days at Hanford, for 115.5 days at Livingston, and for 113.2 days at Virgo. The actual analyzed data amounts are 93.4 days for $\mathrm{HL}, 17.8$ days for $\mathrm{HV}$, and 14.8 days for the $\mathrm{LV}$ network.

The calibration uncertainties for the LIGO detectors in the $20-2000 \mathrm{~Hz}$ frequency range are $<7 \%$ in amplitude, $<4^{\circ}$ in phase, $<1 \mu$ s in timing for O3a [16], and $<12 \%$ in amplitude, $<10^{\circ}$ in phase, $<1 \mu \mathrm{s}$ in timing for $\mathrm{O} 3 \mathrm{~b}$ [17]. The calibration uncertainties for Virgo in most of the $20-2000 \mathrm{~Hz}$ frequency range are $<5 \%$ in amplitude, $<2^{\circ}$ in phase, and $<10 \mu \mathrm{s}$ in timing for all of $\mathrm{O} 3[18,19]$. These uncertainties are not expected to have a significant impact on the search presented here. However, they can contribute to the systematic uncertainties associated with the efficiency numbers quoted in Sec. IV. The O3a GW strain data used in this paper are part of the O3a data release through the Gravitational Wave Open Science Center [22], and can be found at [23].

\section{B. Data quality}

The LIGO and Virgo detectors are affected by various sources of terrestrial noise that can interfere with the detection of GWs [24,25]. In addition to the primary channel recording GWs, the interferometers use a large number of auxiliary channels that observe either the external environment [26,27], or the interferometer itself. Through the use of auxiliary channels, it is possible to substantially reduce the impact of noise transients by discarding (vetoing) a small percentage of observing time during which noise contamination is present [28]. A brief discussion of some of the most relevant data quality issues is presented in this section, but much more detail on these issues and their mitigation can be found in Ref. [20].

To address specific data quality problems, tens of different data quality vetoes defining times to be removed from the search are constructed and applied to the analyses described in this paper. The most significant data quality issues successfully discarded by these vetoes are high signal-to-noise ratio (SNR) glitches associated with light intensity dips in both LIGO interferometers, radio frequency beatnotes (also known as whistles), and a single half-hour period of high amplitude violin mode resonances of the LIGO Hanford suspension system. The effectiveness of each data quality veto is determined based on the ratio of the percentage of glitches removed to amount of observation time vetoed. An additional stage of automated statistical vetoes using the hveto [29] algorithm is subsequently applied using the same procedure as in $\mathrm{O} 2$ [13]. Hveto uses a hierarchical method to produce a ranked list of statistically significant vetoes generated by applying a specific list of SNR thresholds and time windows to a subset of LIGO's auxiliary channels. 
Between $1 \%$ and $2 \%$ of the total observation time per interferometer is discarded due to data quality issues, with precise breakdowns provided in Ref. [20]. A complete list of vetoes used in this search with brief descriptions of each is given in Ref. [30].

Unfortunately, these vetoes do not suppress all nonastrophysical features of the data. As interferometer sensitivity has improved, light scattering has become more prominent at low frequencies $[31,32]$. Scattering noise was significantly reduced in the latter part of the run, but it remained a prominent feature throughout much of $\mathrm{O} 3$, especially during periods of high anthropogenic or seismic activity. Because of the large amount of time with light scattering present and the lack of straightforward and consistent auxiliary channel witnesses, most light-scattering glitches are not vetoed.

Another prominent noise type that is not vetoed by standard methods are blip glitches [33]. These have recurred in both LIGO interferometers throughout the advanced detector era. Blips are short-duration, low quality factor $(Q)$ glitches which occur at the rate of several per day. As these blips do not have clear auxiliary witnesses or known origin, and are not clearly morphologically distinct from some astrophysical models of interest, they must be handled by the search algorithms themselves. During O3 a new population of loud singlepulse bliplike glitches was observed. The origin of these glitches is not known. See Sec. III A 1 for more details on the handling of this glitch class.

\section{UNMODELED GW TRANSIENT ANALYSES}

Using the three-detector HLV network generally enables a more accurate reconstruction of both the structure of the GW signal and its sky location than is possible with a two-detector network. However, for purposes of detection, the sensitivity of the HLV network is not better than the HL network for the $\mathrm{O} 3$ analyses described in this paper. The generic all-sky search for GW bursts cannot rely on assumptions about the GW polarization state. Since the two LIGO interferometers are nearly coaligned and therefore sensitive to similar linear combinations of the GW polarization components over most sky directions, Hanford and Livingston generally detect a given GW with comparable SNRs. Virgo, by contrast, typically senses a different linear combination of GW polarizations. In $\mathrm{O} 3$ the LIGO interferometers have better sensitivity than Virgo (see Fig. 1), and for many source directions the difference in detector orientation enhances this disparity.

In addition, there is a negligible loss in detection efficiency when narrowing the analysis of HL-only data to search for the GW polarization projection that best matches the network from each sky direction. This allows us to implement stricter requirements on the signal coherence between the Hanford and Livingston detectors and results in a more effective rejection of noise transients.
This advantage is not shared by analyses of networks involving Virgo due to its misalignment with the LIGO detectors. To make full use of Virgo data, the analysis has to either open the search to both GW polarization components over the sky, or relax the requirements on the signal coherence between participating detectors. The distribution of non-Gaussian noise outliers present in all detectors in $\mathrm{O} 3$ is thus more effectively mitigated in coherent analyses of the HL network than in analyses with networks including Virgo, and this affects the resulting detection efficiency. The analyses described in this paper therefore use the HL network rather than HLV because we are interested in maximizing detection probability.

The search for short GW bursts is sensitive to CBC sources, especially binary black hole coalescences [13], and hence a fraction of them are found by the analyses presented here. The discussion of the astrophysical properties and implications of the detected $\mathrm{CBC}$ events is the subject of other LIGO Scientific and Virgo Collaboration catalog papers (see Ref. [3] for O3a results). Search results in this paper initially include GWs from CBCs, but known $\mathrm{CBC}$ events are excised in a subsequent step, and discussion here is limited to candidate events that are not found by targeted searches for such sources.

\section{A. Search algorithms}

In order to make the results of the search more robust, two independently developed search algorithms are deployed: coherent WaveBurst (CWB) and BayesWave (BW). The $\mathrm{CWB}$ algorithm is used to analyze the entire dataset. BW is computationally more intensive, thus it is only used to follow up a subset of the dataset identified by CWB in order to provide a partly independent measurement of the candidates' significance. Both of these algorithms and their results are described below.

\section{Coherent WaveBurst}

Coherent WaveBurst is an algorithm based on the maximum-likelihood-ratio statistic over all sky directions applied to excesses of signal power in the time-frequency domain representation of the strain data from the network of detectors [34-36]. The analysis uses the WilsonDaubechies-Meyer wavelet transform at various timefrequency resolutions [37]. Multiple resolutions allow adaptation of time-frequency characterization to the signal features. Coherent WaveBurst is routinely used in LIGOVirgo searches and reconstruction of GW transients $[13,15]$.

In this work the low and high frequency parts of the parameter space are separately covered by two analyses. The same procedure was also done for $\mathrm{O} 1$ [15] and $\mathrm{O} 2$ [13]. The clusters of wavelets which fall above the noise floor of the detectors and pass the internal thresholds of the pipeline are referred as triggers.

The low-frequency analysis covers the frequency range between 16 and $1024 \mathrm{~Hz}$. Triggers with mean reconstructed 
frequency below 24 and $32 \mathrm{~Hz}$ are rejected for O3a and $\mathrm{O} 3 \mathrm{~b}$, respectively, to avoid contamination from loud and frequent low-frequency glitches. The HV and LV networks are analyzed only when one of the LIGO interferometers is unavailable, i.e., there is no overlap in time of dataset for any of the networks considered and the live times for each network are mutually exclusive.

The requirement on the signal coherence across detectors is implemented as a threshold on the network correlation coefficient (referred to as $c_{c}$ in Ref. [34]), which is the fraction of coherent energy in the network of detectors. Triggers are required to pass $c_{c}$ thresholds of 0.8 for the analysis of the HL network and 0.5 for the HV and LV networks, since Virgo is not coaligned with the two LIGO detectors. These thresholds were chosen to optimize overall performance over the set of signal models listed in Table I

The triggers obtained after passing the frequency rejection and network correlation coefficient threshold are further divided into three different, mutually exclusive bins, referred to as LF1, LF2, and LF3. The choice of the bins is based on the background triggers' morphologies, and the goal is to isolate background triggers that are loud and frequent to a small part of the parameter space. LF1 contains triggers with most of the signal energy confined to a single oscillation. In $\mathrm{O} 3$ a population of such short-duration blip glitches

TABLE I. The $h_{\text {rss }}$ values (in units of $10^{-22} \mathrm{~Hz}^{-1 / 2}$ ) for which $50 \%$ detection efficiency is achieved with an IFAR of 100 years for each of the injected signal morphologies. The SG waveforms reported in this table have circular polarization: " $>40$ " indicates that $50 \%$ detection efficiency is not achieved for the maximum $h_{\text {rss }}$ used in this injection set, and "-" denotes waveforms not analyzed by BW.

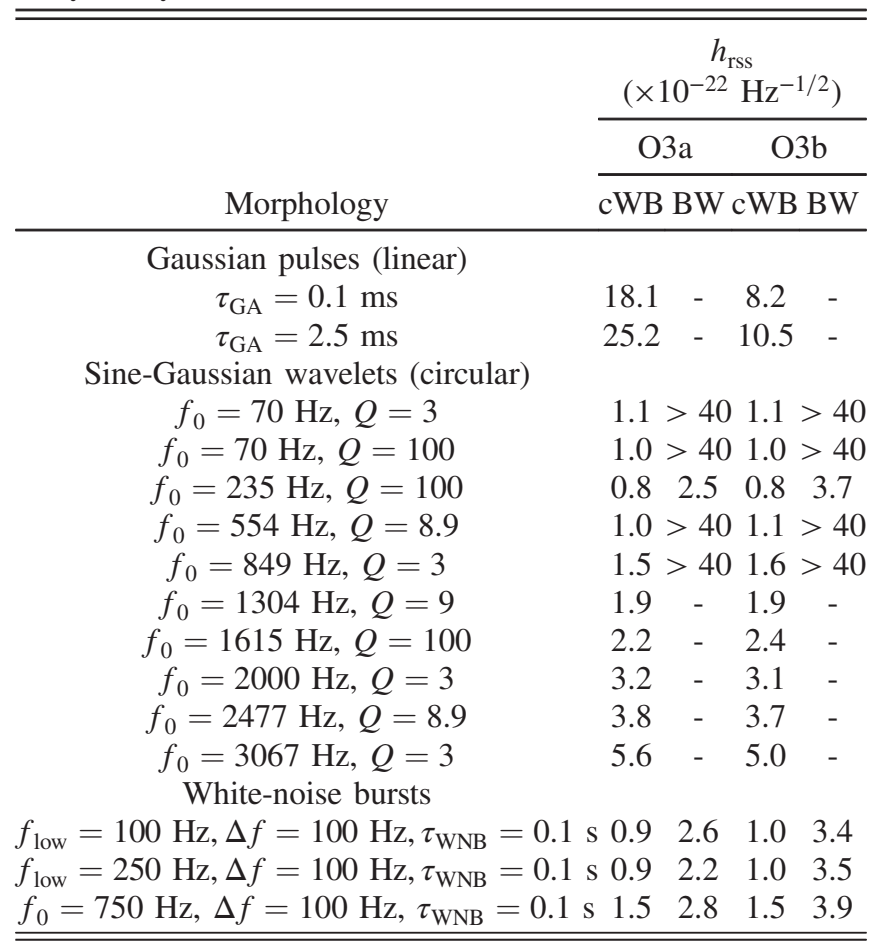

dominates the tail of the background trigger distribution and hence the LF1 bin is introduced in the $\mathrm{O} 3$ search to confine these glitches (see Sec. II B). LF2 contains the remaining triggers that are characterized by $Q \leq 3$, also resembling blip glitches. LF3 contains the higher $Q$ lowfrequency triggers and shows the cleanest background distribution. Unlike $\mathrm{O} 1$ and $\mathrm{O} 2$, nonstationary spectral lines do not contribute significantly to the background in O3.

The low-frequency CWB analysis is performed separately for $\mathrm{O} 3 \mathrm{a}$ and $\mathrm{O} 3 \mathrm{~b}$. The background distribution of triggers is calculated by time-shifting the data of one detector with respect to the other detector by an amount that breaks any correlation between detectors for a real signal. The HL network is time-shifted to obtain a total background live time of around 2000 years. For the HV and LV networks, around 1000 years of background are generated using all coincident data. The use of full coincident time for the HV and LV networks is necessary because the exclusive livetime is not sufficient to produce such large background statistics.

The high-frequency analysis covers the frequency range $1024-4096 \mathrm{~Hz}$. The analysis is carried out in the frequency band $512-4096 \mathrm{~Hz}$ but only triggers with mean reconstructed frequencies $\geq 1 \mathrm{kHz}$ are kept. For this analysis only the HL network is considered, as Virgo is significantly less sensitive than the LIGO interferometers in the high-frequency region (a factor of $\sim 5$ above $1000 \mathrm{~Hz}$, see Fig. 1). Similarly to the low-frequency analysis, a network correlation coefficient threshold of 0.8 is used for the high-frequency part of the analysis. No division of background triggers into analysis bins is required for this analysis. However, during the first part of $\mathrm{O} 3$ run until May 16, 2019, there were background triggers dominating the tail with central frequency $f_{0}>3400 \mathrm{~Hz}$; for this part of the run only the triggers with central frequency $\leq 3400 \mathrm{~Hz}$ are admitted. The full frequency range is considered for all times from May 16 onward. As a result, the high-frequency CWB analysis is divided into three parts, the first two parts are in O3a (before and after May 16, 2019, see above), and the third part corresponds to all of O3b. Total background live times of around 1000 years are produced for $\mathrm{O} 3$.

The significance of each trigger is calculated by comparing the coherent network SNR $\eta_{c}$ [34] with the background distribution of the bin and the network to which the trigger belongs. The inverse false alarm rate (IFAR) is calculated for each observed trigger. The IFAR for the low-frequency analysis is penalized by a trials factor of 3 corresponding to the three analysis bins LF1, LF2, and LF3. The criteria for a significant detection of an event is set at IFAR $\geq 100$ years.

The analysis results for the CWB low-frequency region are shown in Fig. 2. The loudest candidate event in the HL network after excluding known CBCs [3] occurred at UTC 2019-09-28 02:11:45. This candidate has an IFAR of 0.53 years. The second most significant candidate in this network occurred at UTC time 2019-08-04 08:35:43, with an IFAR of 0.19 years. The loudest candidate for the $\mathrm{HV}$ and $\mathrm{LV}$ 

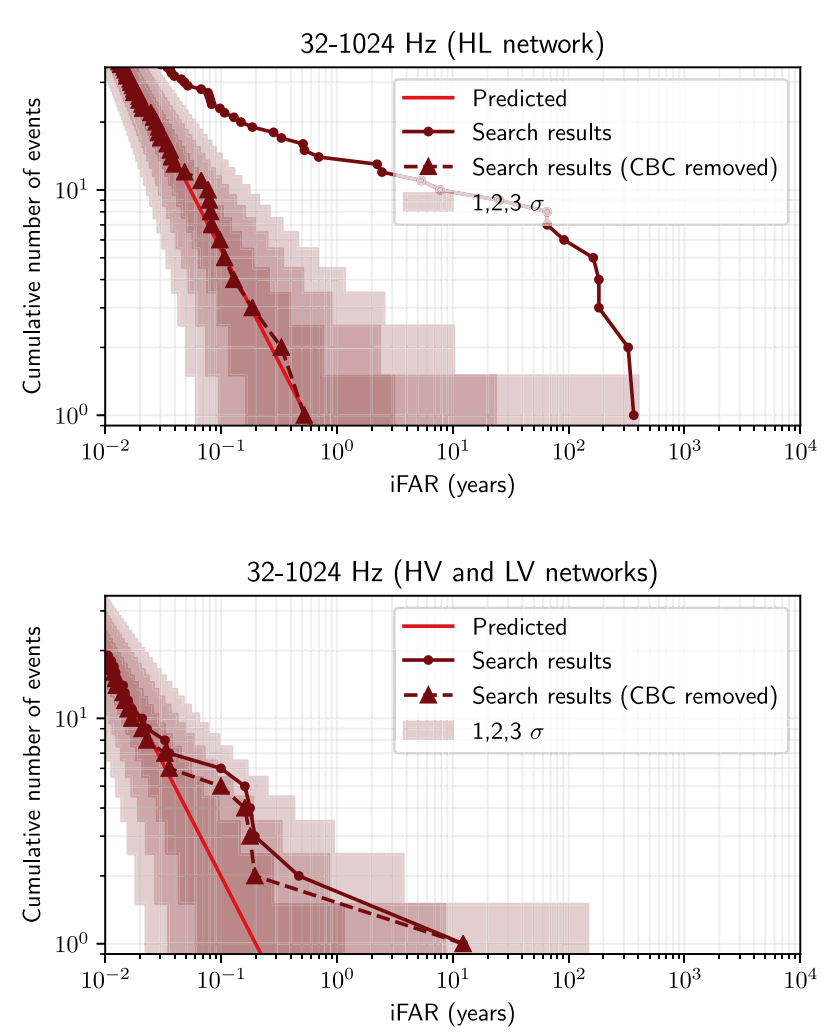

FIG. 2. Cumulative number of events versus IFAR found by the CWB low-frequency analysis using all O3 data for the HL network (top panel), and the HV and LV networks combined (bottom panel). Circular points show results for all data and triangular points show after times around all known compact binary coalescence sources have been excised. The solid line shows the expected mean value of the background, given the analyzed time. The shaded regions show the 1,2 , and $3 \sigma$ Poisson uncertainty regions.

networks is an HV event at UTC time 2019-04-30 00:49:32, with an IFAR of 12 years. Though none of these meet the IFAR threshold of 100 years for a detection, investigations into these loudest remaining candidates are discussed further in Sec. III B.

The results for the high-frequency CWB analysis are shown in Fig. 3, the loudest event has an IFAR of 0.3 years.

\section{BayesWave}

BW [38-40] is a Bayesian algorithm modeling both GW signals and non-Gaussian noise transients as sums of sine-Gaussian wavelets. The number of wavelets used is marginalized over using a transdimensional reversible jump Markov chain Monte Carlo algorithm. The detection statistic used is the natural logarithm of the signal-to-glitch Bayes factor $\left(\ln \mathcal{B}_{\mathcal{S}, \mathcal{G}}\right)$, i.e., the Bayes factor between the signal model consisting of Gaussian noise and an astrophysical signal coherent across detectors; and the glitch model, which describes the data as Gaussian noise and glitches modeled independently in each detector. Thus a positive $\ln \mathcal{B}_{\mathcal{S}, \mathcal{G}}$

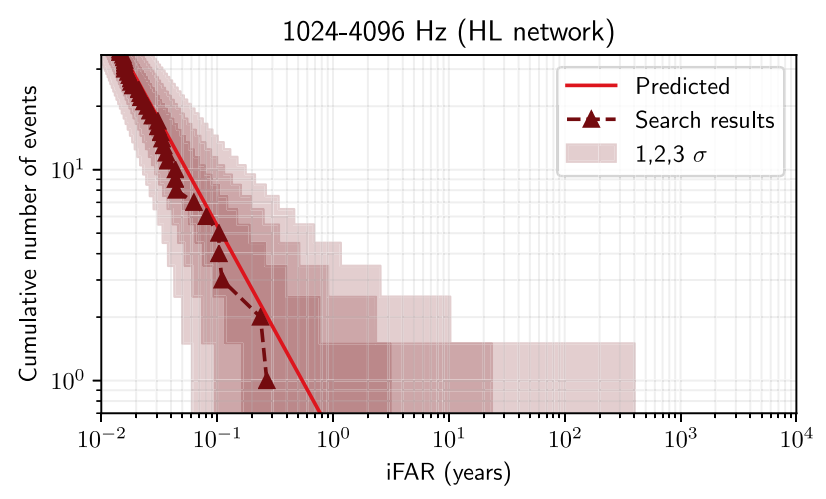

FIG. 3. Cumulative number of events versus IFAR found by the CWB high-frequency analysis (triangular points) using all $\mathrm{O} 3$ data for the HL network (Virgo is not used for high-frequency search). The solid line shows the expected mean value of the background, given the analyzed time. The shaded regions show the 1,2 , and $3 \sigma$ Poisson uncertainty regions.

indicates that the presence of a GW signal is favored, while a negative $\ln \mathcal{B}_{\mathcal{S}, \mathcal{G}}$ shows support for the event being a glitch.

Due to the transdimensional sampling it requires, analyzing the entire $\mathrm{O} 3$ dataset with $\mathrm{BW}$ is computationally prohibitive. Thus BW is used as a follow-up to the CWB pipeline, similarly to previous observing runs $[13,15]$. By doing so an additional measurement of IFAR for the candidates followed up by BW is acquired, thus making the search presented in this paper more robust against potential shortcomings of individual algorithms. BW followed up CWB candidates in the low-frequency analysis, treating all the search bins as a single bin, and using a threshold of $\eta_{c}=9.90$. This is the same threshold as the one used in the $\mathrm{O} 2$ analysis [13], and was chosen as the lowest value that results in a computationally manageable number of background triggers. BW uses the same background dataset as CWB from time slides.

A total of $22 \mathrm{CWB}$ candidates are above the $\eta_{c}$ threshold, 19 of which are known CBC candidate events described in recent or future publications. This is fewer than found by $\mathrm{CWB}$, because not all $\mathrm{CBC}$ candidates passed the $\mathrm{BW}$ follow-up threshold. The combined results from all detector networks are shown in Fig. 4 in terms of the cumulative distribution of their IFAR values. The three candidate events remaining after removing the known $\mathrm{CBC}$ candidate events are discussed in the previous section. None of these is found with high enough significance in $\mathrm{BW}$ to be considered a likely GW event. Section III B discusses these candidate burst events.

\section{B. Candidate events}

\section{Surviving non-CBC candidates}

The three non-CBC candidate events with $\eta_{c}$ values above 9.90, a high enough value to trigger BW follow-up, are discussed individually below. They are identified by the 


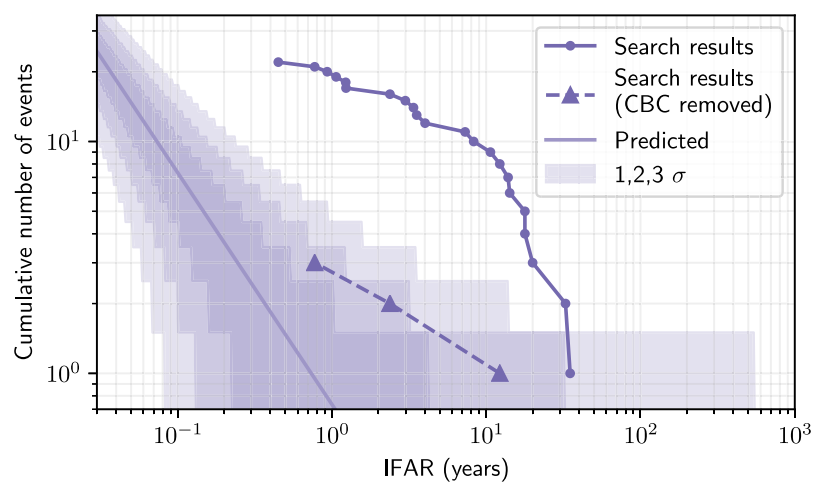

FIG. 4. Cumulative number of events versus IFAR found by the $\mathrm{BW}$ follow-up to the CWB low-frequency analyses using all $\mathrm{O} 3$ data (circular points) and after times around all compact binary coalescence sources have been excised (triangular points). The solid line shows the expected background, given the analyzed time. The shaded regions show the 1,2 , and $3 \sigma$ Poisson uncertainty regions.

time at which they occurred. In each case, the statistical significance is not high enough to claim the candidate as a GW event. Though none of these candidates are vetoed by data quality procedures, data quality concerns for each case are discussed.

2019-09-28 02:11:45 UTC: the most significant HL CWB candidate has an inverse false alarm rate of 0.53 years in a CWB all-sky search and 0.8 years in the BW follow-up. This initially appeared in the low-latency CBC-focused CWB analysis but was manually rejected in near-real time as most probably being caused by a glitch in the Livingston detector [41]. It does not pass signal consistency cuts specific to the version of that search focused on CBCs, described in Ref. [3], but remains in the more general burst analysis at lower significance. Instrumental investigations into possible origins focused on magnetic noise in the station at the end of Hanford's X arm, but magnetic coupling was ruled out as a significant contributor to the power of the putative signal. The morphology in the Livingston detector resembles Tomte glitches [24,42] appearing at other times, while there is little power in the Hanford detector. The significant difference in the relative amplitude between Hanford and Livingston would mean that, if astrophysical, this candidate event would have to originate from the $\sim 5 \%$ of the sky where Hanford has negligible sensitivity but Livingston's sensitivity is significant.

2019-08-04 08:35:43 UTC: the second most significant low-frequency HL CWB candidate, at an IFAR of 0.19 years, was also initially identified in a low-latency CWB targeted search for binary black hole coalescences, but it did not meet the significance threshold to generate a public alert. BW follow-up finds an IFAR of 12.2 years, making this the most significant non-CBC outlier in that analysis. It occurred less than a second after an SNR $\sim 60$ series of glitches in Livingston, which are themselves too loud to be astrophysical. These glitches morphologically resemble the repeating blips class of glitches [42] occurring at other times in both LIGO interferometers. Its close proximity to these glitches makes it impossible to discount an instrumental origin, though it is not vetoed by any auxiliary witness channel. As a follow-up study to the low-latency search, BW was used to model the glitches occurring just before the candidate event, and that model was subtracted from the data in order to produce a deglitched data stream [40]. It was found that this glitch subtraction lowered the SNR but had negligible effect on the reconstructed morphology of the candidate.

2019-04-30 00:49:32 UTC: an additional candidate is identified in the HV O3a CWB search, a less sensitive network than HL, at an IFAR of 12.29 years. It was not identified as a trigger of interest in the low-latency search. The BW follow-up gives an IFAR of 2.4 years for this trigger. The presence of blip glitches in Hanford less than a second prior to the candidate and the resemblance to a blip glitch in the Hanford interferometer lead to similar data quality concerns as the previous trigger.

\section{Low-latency-only candidates}

In the low-latency search described in Sec. I, public alerts were generated for burst search candidates with significance exceeding an IFAR of 4 years. Two candidate events crossed this significance threshold in the low-latency CWB search but do not appear in the version of the analysis presented in this paper, as explained below.

S191110af: this was a high-frequency $(\sim 1780 \mathrm{~Hz}) \mathrm{HL}$ CWB candidate that generated a public alert [43] based on its significance in the low-latency CWB analysis. Follow-up of the candidate shortly after it was identified indicated that it was due to a faulty piezoelectric transducer at Hanford. This candidate event does not appear in the analysis described in this paper, as times strongly affected by this noise were vetoed [20]. It is no longer of astrophysical interest.

S200114f: this HL candidate generated a public alert [44] based on its significance in the low-latency unmodeled CWB all-sky search, but it is not found in the analysis as described in this paper because it fails an internal CWB consistency cut (the network correlation coefficient $c_{c}<0.8$, see Sec. III A 1) requiring the signal to be correlated between the two LIGO detectors. It is further discussed in the $\mathrm{O} 3$ intermediate mass black hole search paper [4].

\section{ASTROPHYSICAL INTERPRETATION OF THE RESULTS}

In order to place the search results in an astrophysical context, it is necessary to measure detection efficiency for plausible signals. This is accomplished by injecting simulated signals (via software) into the detector data and recovering them using the search methods described in previous 
sections. The pipelines' abilities to recover a broad range of transient signals can be tested by this method. These transient signals include a set of ad hoc waveforms as well as astrophysically motivated waveforms from CCSNe and neutron star $f$ modes. The sensitivity of the search to these simulated signals is described in this section. Only the HL network is used for quoting sensitivities, as the other network pairs provide sensitivities which are at least a factor of 2 worse in amplitude. The sensitivities quoted in this section follow the criterion for significant detection of IFAR $\geq$ 100 years.

\section{A. Sensitivity to generic signal morphologies}

As the pipelines are able to detect GWs from a range of potential astrophysical sources, a set of ad hoc waveforms comprising a wide range of different morphologies are used to estimate the search sensitivity to generic signals. The waveform families used here are sine-Gaussian wavelets (SG), Gaussian pulses (GA), and band-limited white-noise bursts (WNB). The SG signals are defined by the central frequency $f_{0}$ and quality factor $Q$, which determine the signal's duration. The GA signals are described by the duration of one standard deviation $\tau_{\mathrm{GA}}$. The WNB signals are described by their lower frequency bound $f_{\text {low }}$, bandwidth $\Delta f$, and duration $\tau_{\mathrm{WNB}}$. Further details on these waveform morphologies can be found in the S6 short duration all-sky search [45]. These ad hoc signals are injected in the network of detectors over a range of amplitudes, which are expressed in terms of the root-mean-squared strain amplitude $\left(h_{\mathrm{rss}}\right)$ given by

$$
h_{\mathrm{rss}}=\sqrt{\int_{-\infty}^{\infty}\left(h_{+}^{2}(t)+h_{\times}^{2}(t)\right) \mathrm{d} t},
$$

where $h_{+}$and $h_{\times}$are the components of the signal polarizations in the source frame.

There are differences in the distribution of extrinsic parameters for the SG and GA injections with respect to the $\mathrm{O} 2$ search [13]. For the $\mathrm{SG}$ and GA waveforms in $\mathrm{O} 3$ the simulated signals are injected over a grid of maximum strain values given by $h_{\mathrm{rss}}=(\sqrt{3})^{N} 5 \times 10^{-23} \mathrm{~Hz}^{-1 / 2}$, where $N$ ranges from 0 to 8 . The strain distribution for the $\mathrm{O} 2$ search was uniform in the square of the signal distance. Similarly to O2, the simulated signal sources are drawn from a uniform distribution in solid angle over the sky. The polarization for GA waveforms is linear, whereas the SG waveforms use both elliptical SG, which are uniform in cosine of the inclination angle of the source, and circular SG, which assume an optimally oriented source. The inclination angle is defined by the angle between the total angular momentum vector and the line of sight. In order to have a direct comparison of sensitivity between the observing runs, the same set of WNB waveforms as described in Ref. [13] are injected into O3 data.
The $h_{\text {rss }}$ values at which $50 \%$ of signals are detected with an IFAR $\geq 100$ years for each waveform are given in Table I. Calibration uncertainities affect the results to at most $10 \%$ as discussed in Sec. II A. Results for the SG waveforms are given only for the circular SG, which is the best case scenario. The results show an imbalance in the sensitivity of the CWB and BW pipelines for SG waveforms. This is due to the fact that the detection statistic $\ln \mathcal{B}_{\mathcal{S}, \mathcal{G}}$ used by BW scales linearly with the number of wavelets used in the reconstruction $[46,47]$. Because SG and GA waveforms can be accurately reconstructed using a single wavelet, $\mathrm{BW}$ is less sensitive to these particular signals. For O3 the sensitivity to GA is worse compared to $\mathrm{O} 2$, this is mainly due to the population of blip glitches during $\mathrm{O} 3$ that resembled GA injections, and are isolated in a dedicated bin as described in Sec. III A 1.

The detection efficiencies obtained can be used to determine the typical amount of energy emitted in GWs needed for a detection. This is done assuming a standardsiren source at a distance of $r_{0}=10 \mathrm{kpc}$ radiating GWs isotropically at a central frequency of $f_{0}$. The amount of energy radiated assuming the signal to be narrow band is then given by Ref. [45]

$$
E_{\mathrm{GW}}^{\mathrm{iso}}=\frac{\pi^{2} c^{3}}{G} r_{0}^{2} f_{0}^{2} h_{\mathrm{rss}}^{2} .
$$

This equation is valid for circular SG and WNB injections, while for the case of elliptical SG injections the energy is given as $E_{\mathrm{GW}}^{\mathrm{rot}}=(2 / 5) \times E_{\mathrm{GW}}^{\text {iso }}$, accounting for the rotating system emission [48]. The narrow band approximation used in this equation leads to $\leq 6 \%$ systematics in computed energy for WNB and is much lower $(\leq 3 \%)$ for the SG injections. This approximation does not hold for the GA injections, which are broadband. The $h_{\mathrm{rss}}$ values for $50 \%$ detection efficiency are used to find the typical amount of energy that needs to be radiated by the GW source in order to be detected by CWB. These results are shown in Fig. 5. The WNB injections for $\mathrm{O} 2$ are carried forward for comparison with O3. WNB results show a factor of 2 improvement, compatible with improvements in detector sensitivity.

Given that the searches do not find any GW transient sources beyond the known CBC signals, the upper limit of the rate per unit volume of non-CBC standard-siren sources [45] has been updated, as shown in Fig. 6. These upper limits use the elliptical SG and WNB injection sets as representative morphologies for non-CBC GW bursts. The markers represent the upper limit for rate density at $90 \%$ confidence [45], calculated at an IFAR $\geq 100$ years. The results shown in Fig. 6 assume that $1 M_{\odot} c^{2}$ of GW energy has been emitted from the source. The upper limits can be scaled to any emission energy $E_{\mathrm{GW}}$ by using Eq. (2) to find that the rate-density scales as $\propto E_{\mathrm{GW}}^{-3 / 2}$. O3 


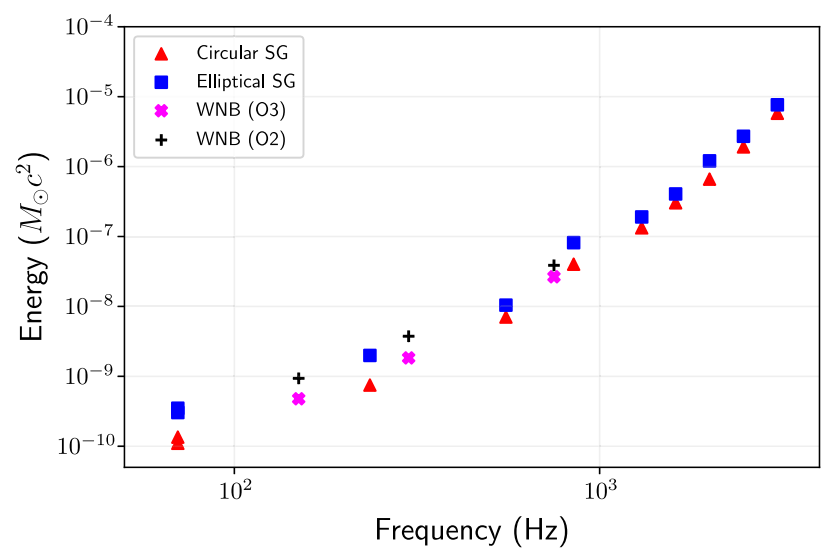

FIG. 5. The GW emitted energy in units of solar masses that correspond to a $50 \%$ detection efficiency at an IFAR of $\geq 100$ years, for a source emitting at $10 \mathrm{kpc}$. The wave forms represented here include all of the circular SG and WNB injections as given in Table I using $E_{\mathrm{GW}}=E_{\mathrm{GW}}^{\text {iso }}$. The SG waveforms with uniform distribution in cosine of inclination angle (elliptical $\mathrm{SG}$ ) are also reported using $E_{\mathrm{GW}}=E_{\mathrm{GW}}^{\mathrm{rot}}$. Only CWB results are presented for $\mathrm{O} 3$ as it is the most sensitive pipeline for the injection set used here. The same results for $\mathrm{O} 2$ are also shown for comparison for the WNB waveforms.

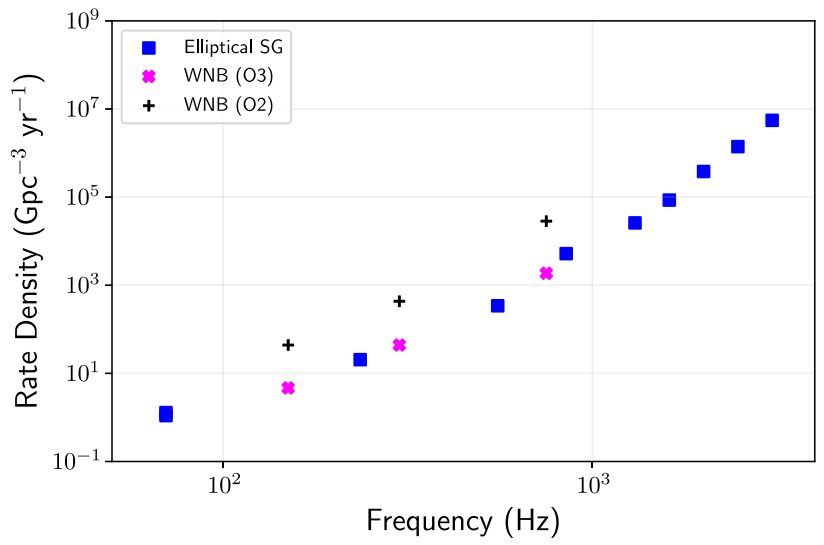

FIG. 6. Upper limits for the GW rate density at $90 \%$ confidence intervals as measured for the $\mathrm{O} 3 \mathrm{CWB}$ analysis using the elliptical SG and WNB waveforms are plotted assuming $1 M_{\odot} c^{2}$ of $\mathrm{GW}$ energy has been emitted from the source. For WNB waveforms the results from $\mathrm{O} 2$ are also plotted for comparison: the $\mathrm{O} 3$ rate density upper limit is about one order of magnitude better than that achieved in O2. These results can be scaled to any emission energy $E_{\mathrm{GW}}$ in solar masses using the relation rate density $\propto E_{\mathrm{GW}}^{-3 / 2}$.

results show about an order of magnitude improvement with respect to $\mathrm{O} 2$ for the WNB injections. The improvement in rate upper limits with respect to $\mathrm{O} 2$ is attributed to a combination of more sensitive detectors, improved pipelines, and the longer duration of the $\mathrm{O} 3$ run.

\section{B. Sensitivity to CCSNe}

Observing GWs from a CCSN would provide invaluable insight into the dynamics of these sources (e.g., [49]). Past searches have looked for GWs in close spatial and temporal proximity to electromagnetically (EM) observed CCSNe within approximately $20 \mathrm{Mpc}[7,50]$. It might also be possible to detect GWs from a CCSN even if its EM signatures cannot be observed, e.g., due to extinction along the line of sight, or in case of a failed supernova [51]. Since the low-frequency unmodeled burst search presented in this paper looks for GW signals in the frequency range relevant to the majority of CCSNe, and their signal can show complex time-frequency structure, it is worthwhile to investigate the sensitivity of this search to GWs from CCSNe. The feasibility of detecting and reconstructing GWs from the next Galactic CCSN event in the upcoming observing runs are extensively studied in Ref. [52].

Sensitivity to CCSNe is tested by analyzing waveforms from five different three-dimensional CCSN simulations. The first three represent typical CCSNe:

(i) Model s18 [53] has a solar-metallicity nonrotating progenitor with a zero age main sequence (ZAMS) mass of $18 M_{\odot}$. The GW emission shows the typical rise in frequency with time associated with the protoneutron star $g$-mode excitation. The peak GW amplitudes occur shortly after shock revival at frequencies in the range of 800-1000 Hz.

(ii) Model $m 20$ (mesa20_3D_pert from Ref. [54]) also corresponds to a solar-metallicity nonrotating progenitor, but it has a higher ZAMS mass of $20 M_{\odot}$ and uses different modeling techniques. The GW emission shows the typical $g$-mode frequency rise, reaching $\sim 1100 \mathrm{~Hz}$ at the end of the simulation. Standing accretion shock instabilities [55-57] leave a subdominant imprint at frequencies of $50-100 \mathrm{~Hz}$, slowly increasing in time.

(iii) Model $s 9$ [58] has a solar-metallicity nonrotating progenitor with ZAMS mass of $9 M_{\odot}$. Due to its mass being in the low end of CCSNe progenitors, the density decreases rapidly outside the core and the model explodes shortly after bounce $(\sim 0.2 \mathrm{~s})$. The GW signal shows the typical $g$-mode pattern with rising frequency and highest amplitudes within the first $\sim 0.35 \mathrm{~s}$ postbounce, reaching $\sim 700 \mathrm{~Hz}$.

In addition to these three models describing typical CCSNe, two simulations corresponding to more extreme CCSNe are also considered. These have higher GW amplitudes but also lower expected rates compared to typical CCSNe (e.g., [59,60]):

(iv) Model $m 39$ [61] describes a CCSN with a massive and rapidly rotating Wolf-Rayet star progenitor with a helium star mass of $39 M_{\odot}$, a metallicity of 1/50 solar metallicity, and an initial surface rotation velocity of $600 \mathrm{~km} \mathrm{~s}^{-1}$. The rapid rotation results in larger GW 
amplitudes. At around $0.4 \mathrm{~s}$ after core bounce, the GW amplitude peaks at a frequency of $\sim 750 \mathrm{~Hz}$.

(v) Model 35OC (35OC-RO from [62]) is a simulation where the explosion is driven by strong magnetic fields and rapid rotation. The progenitor is a subsolar metallicity star with ZAMS mass of $35 M_{\odot}$ and equatorial surface rotation velocity of $380 \mathrm{~km} \mathrm{~s}^{-1}$, evolved with rotation and magnetic fields. Its high rotational energy leads to a strongly oblate shape. The waveform includes the bounce signal and oscillations above $100 \mathrm{~Hz}$.

These five waveforms are chosen to represent the main physical phenomena involved and different modeling methods used.

Using each of the five CCSN models, 1000 waveforms with a uniform-in-distance distribution are generated. The maximum distance for these injections is set to $D=$ $[25,5,5,70,70] \mathrm{kpc}$ for the $s 18, m 20, s 9, m 39$, and $350 C$ models, respectively. All other extrinsic parameters (sky coordinates, source orientation, polarization angles) are randomized, using uniform distributions covering the full ranges of physically possible values. The sets of 1000 waveforms are repeated multiple times to cover the whole duration of the observing run.

Results from analyzing the injections with $\mathrm{BW}$ and $\mathrm{CWB}$ are shown in Fig. 7 as distances at which $50 \%$ or $10 \%$ of injected signals are detected, using the same IFAR threshold of 100 years as in Sec. IVA. The figure shows that waveforms corresponding to typical CCSNe are generally detectable only within a few kiloparsecs, while CCSNe which produce higher GW amplitudes can be observed out to tens of kiloparsecs. The CWB algorithm can detect all waveforms at similar, but slightly larger distances than BW. The largest distance at which $10 \%$ efficiency is reached is $40.7 \mathrm{kpc}$ (CWB for the 35OC model), which is smaller than the typical range of currently operating neutrino detectors (e.g., [63]).

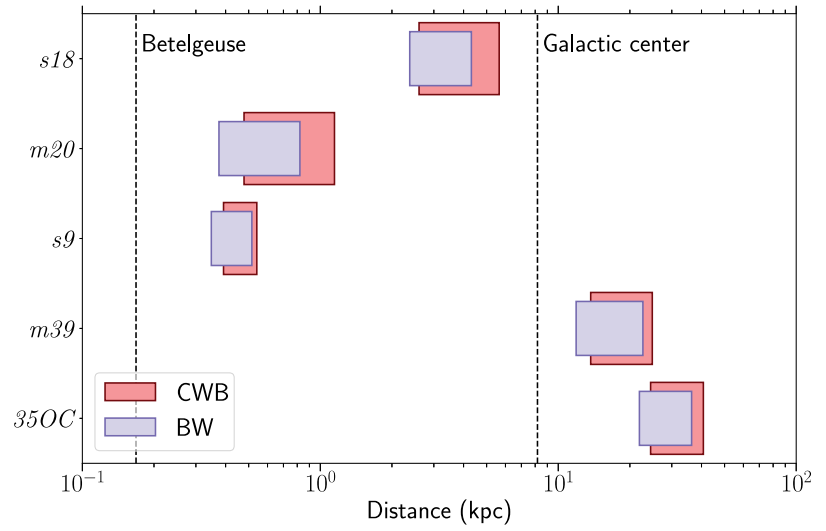

FIG. 7. Distances at which $50 \%$ and $10 \%$ detection efficiencies are reached for different CCSN waveforms indicated by the left sides and right sides of rectangles, respectively. Different colors represent results from the two detection algorithms used.
TABLE II. The overall efficiency values with an IFAR of 100 years for each of the injected CCSN waveforms. There is a significant difference in efficiency between models of typical CCSNe and those with higher GW amplitudes. For two of the typical CCSN models ( $m 20$ and $s 9$ ) the efficiency is practically zero. This is due to the fact that these can only be detected out to $\sim 1 \mathrm{kpc}$, while the Galactic distribution provides few CCSNe at such a close distance.

\begin{tabular}{lccccc}
\hline \hline Model & $s 18$ & $m 20$ & $s 9$ & $m 39$ & $350 C$ \\
\hline cWB & $1.2 \%$ & $<0.1 \%$ & $<0.1 \%$ & $69.4 \%$ & $89.8 \%$ \\
BW & $0.3 \%$ & $<0.1 \%$ & $<0.1 \%$ & $65.4 \%$ & $89.1 \%$ \\
\hline \hline
\end{tabular}

Thus any CCSN detection by the search presented in this paper would have an observable neutrino counterpart.

The same waveforms are also generated with a spatial distribution sampling the stellar mass distribution of the Milky Way, which is modeled as consisting of a bulge, a thick stellar disk and a thin stellar disk, with parameters taken from $[64,65]$. The overall efficiency for these injection sets is reported in Table II. These represent the total fraction of simulated signals recovered, and thus are indicative of the probability that the search presented in this paper would detect a Galactic CCSN event given that the detectors were operational and under the assumption of a given CCSN model. For two typical CCSN models ( $m 20$ and $s 9$ ) the search did not detect any of the simulated signals, so an upper limit on the efficiency is quoted. This is expected, since the detector network is only sensitive to these waveforms out to $\sim 1 \mathrm{kpc}$, and the Galactic matter density model is strongly peaked around the Galactic center, so there are very few simulated signals at small distances. BW and CWB achieve low efficiencies for $s 18$, while they both detect a large fraction of events from the two models producing higher $\mathrm{GW}$ amplitudes ( $m 39$ and 35OC).

\section{Sensitivity to isolated neutron star emitters}

A fraction of the neutron star population is known to show transient excitations measured by EM observations. These involve glitches from pulsars, as well as glitches and flaring activities from magnetars. The observed rate of such phenomena is expected to be accompanied by a larger rate of yet unobserved events. This work focuses only on glitches, since a dedicated search for the case of magnetar bursts is performed by a dedicated search (see Ref. [66] for O2 results). The two most-explored mechanisms in the literature for these neutron star excitations are starquakes and superfluid crust interactions [67]. In the superfluid mechanism there is an interaction of internal superfluid with the solid crust of a neutron star $[68,69]$. Because of superfluid vortex avalanches during the spin-up phase of pulsar glitches, the excitation of one or more families of global oscillations in the neutron star leads to a GW signal on a time scale around $40 \mathrm{~s}$ before the observed jump in frequency. A search for short 
transient GW emission associated with oscillations of the fundamental quadrupole mode excited by a pulsar timing glitch was performed with the data from LIGO's fifth science run (S5). No GW detection candidate was found associated with a timing glitch in the Vela pulsar in August 2006 and a Bayesian $90 \%$ credible upper limit of $6.3 \times 10^{-21}$ on the peak intrinsic strain amplitude of $\mathrm{GW}$ assuming a ring-down signal was set [9].

The precise model of the short-duration GW burst signal depends upon various considerations about the internal mechanism of the angular momentum transfer. The bulk emission of GW bursts is assumed to be due to $f$ mode excitation $[9,70]$. Here it is assumed that the GW burst signal coming from the glitching neutron star is completely described by the f-mode oscillation modeled by a damped sinusoid and the optimistic scenario of the total glitch energy being converted to GW energy, $E_{\text {glitch }}=E_{\mathrm{GW}}$. The same approach was followed in previous studies [9].

Estimates of the frequency and damping time of the neutron star fundamental quadrupole mode for various models of the equation of state (EOS) indicate that the related GW frequency is expected in the range $2 \mathrm{kHz} \leq$ $\nu_{\mathrm{GW}} \leq 3 \mathrm{kHz}$ and the damping time is in the range of tens of milliseconds to as much as half a second [70]. Hence, the higher frequency part of the HL all-sky search for generic bursts can survey these phenomena and motivates a dedicated astrophysical interpretation to explain the search's reach and coverage of Galactic sources.

The following discussion focuses on providing the sensitivity of the all-sky search for GWs arising from neutron star glitches. Here the Vela pulsar is used as a standard siren (distance of $287 \mathrm{pc}$ and $\operatorname{spin} \nu_{s}=11.2 \mathrm{~Hz}$ ) to interpret the results as it is the closest known glitching pulsar [71,72]. The signal injections are uniform in all-sky directions and the source is assumed to be optimally oriented, i.e., circularly polarized. The $f$-mode damped sinusoid's frequencies and damping times are related to the mass and radius of isolated neutron stars in the nonrotating limit [73]. The neutron star masses are in the range of 1-2 $M_{\odot}$ with $0.25 M_{\odot}$ bins. The radius of the neutron star for each mass bin is determined by using two EOS, these are APR4 (soft) [74] and H4 (hard) [75]. The observation of GW170817 suggests that APR4 is preferred over H4 [76,77]. The sensitivity is determined using the $h_{\text {rss }}$ values at $50 \%$ detection efficiency for each mass bin and EOS. From this the detectable glitch size $\Delta \nu_{s}$ is determined using Eq. (5) in Ref. [70], assuming that the neutron star has the same distance and spin as the Vela pulsar. The typical $h_{\mathrm{rss}}$ at $50 \%$ detection efficiency for an IFAR of 100 years is around $10^{-22} \mathrm{~Hz}^{-1 / 2}$. The sensitivities are reported in terms of glitch size as a function of mass and EOS in Fig. 8. The detectable glitch size for the $\mathrm{O} 3$ run is around $10^{-4} \mathrm{~Hz}$, whereas the actual glitch sizes vary between $10^{-9}$ and $10^{-4} \mathrm{~Hz}$ [78-80]. The sensitivities obtained for $\mathrm{O} 3$ are thus not in the range where a detection would be expected.

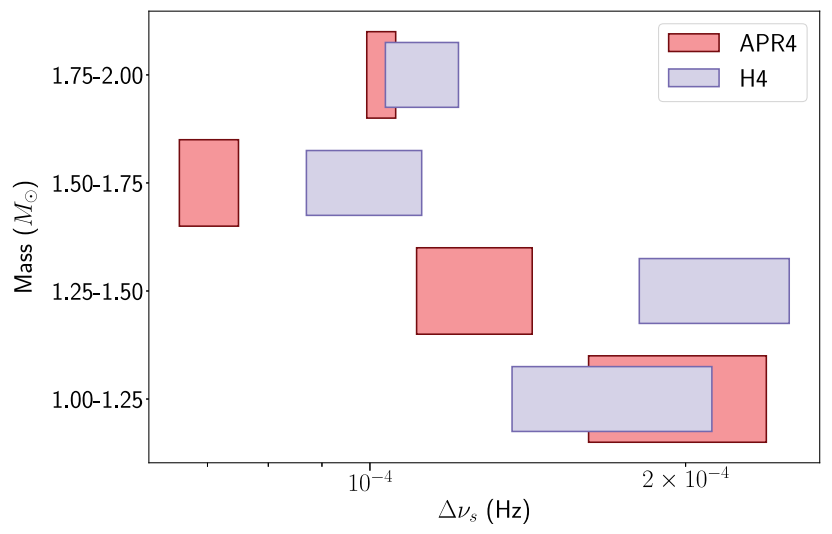

FIG. 8. Sensitivity to neutron star glitches is shown in terms of detectable glitch size by considering the Vela pulsar as a standard siren (distance and spin of Vela) for soft (APR4) and hard (H4) EOS assuming an optimally oriented source. For each EOS the boxes show the search sensitivity of the glitch size for $50 \%$ detection efficiency at IFAR $\geq 100$ years, and the spread of the box shows the variation within the mass bin. A higher-mass neutron star allows for smaller glitches to be detected. Glitch size across the parameter space for a Vela-like pulsar would need to be stronger than $\sim 10^{-4}$ for $50 \%$ of the sources to be detected in $\mathrm{O} 3$.

\section{CONCLUSION}

This paper reports the results of a search for short duration GW transients of generic morphologies in O3. The search uses minimal assumptions on the signal waveform, direction and arrival time and targets bursts with duration up to a few seconds with reconstructed central frequency from 24 to $4096 \mathrm{~Hz}$. The CWB algorithm provides results for the entire frequency range, while the BW algorithm performs a followup of the loudest CWB candidate events with frequencies up to $1 \mathrm{kHz}$. Both analyses detect GWs from CBC which have been identified by other targeted analyses for these sources. These detections are not discussed in this paper and instead have been included in papers dedicated to CBCs [3], or will be included in upcoming papers. No other significant events have been found. The three loudest candidates remaining in the search are discussed, but their statistical significance is insufficient to exclude an instrumental origin. Two unmodeled GW transient candidates that triggered online public alerts are also discussed, with explanations of why they do not appear in this search.

The null result of this search allows setting of rate-density upper limits, similarly to what was done for previous observation runs $[13,15,45]$ at an inverse false alarm rate threshold of 100 years. The current upper limit is about one order of magnitude better than the previous $\mathrm{O} 2$ limit over most of the frequency bandwidth [13], mainly due to improved spectral sensitivity of the detectors and increased observation time. In addition, the typical sensitivity of this search improves by about two orders of magnitude at the lowest frequencies tested $(70 \mathrm{~Hz})$. The latter result stems from 
a combination of lower detector noise, better cleaning of data from power line sidebands, and algorithm improvements for glitch classification. The null results can be used to estimate sensitivity to certain classes of GW signals: CCSNe and isolated NS excitations. No specific tuning of the analysis is attempted, in order to preserve the general character of the search. Five CCSN models have been tested: for the two models that produce higher GW amplitudes, the coverage of the Galaxy by this search is already good for the $\mathrm{O} 3$ search. However, for more typical CCSN models, the current coverage of the Galaxy is still poor. It is expected that during the next observation runs some of these, e.g., model $s 18$, might also achieve good Galactic coverage using GW information alone, while the distance at which CCSNe described by models producing higher GW amplitudes are detectable could reach the distance of nearby dwarf galaxies, like the Large Magellanic Cloud. The neutron star signals considered are $f$-mode emissions, modeled as damped oscillations with central frequency and damping time determined by two equations of state for the stellar mass range $1-2 M_{\odot}$. The sensitivities achieved by this search for generic bursts are still not sufficient to be able to detect such high-frequency transients at the energy scale of pulsar glitches from, e.g., the Vela pulsar at high confidence. Nevertheless the outlook is promising, since the expected improvements of the GW detectors in the high-frequency band for the next observation run are quite relevant [81], e.g., a factor of 4 and 2 in amplitude strain spectral density for Virgo and LIGO Hanford, respectively. The resulting improvement on the detectable glitch size is quadratic, so near future untargeted all-sky searches for GW bursts will start probing the physical energy range observed in Vela pulsar glitches.

This article has been assigned the LIGO document number P2100045.

\section{ACKNOWLEDGMENTS}

This material is based upon work supported by NSF's LIGO Laboratory which is a major facility fully funded by the National Science Foundation. The authors also gratefully acknowledge the support of Science and Technology Facilities Council (STFC) of the United Kingdom, the MaxPlanck-Society (MPS), and the State of Niedersachsen/ Germany for support of the construction of Advanced LIGO and construction and operation of the GEO600 detector. Additional support for Advanced LIGO was provided by the Australian Research Council. The authors gratefully acknowledge the Italian Istituto Nazionale di Fisica Nucleare (INFN), the French Centre National de la Recherche Scientifique (CNRS) and the Netherlands Organization for Scientific Research, for the construction and operation of the Virgo detector and the creation and support of the EGO consortium. The authors also gratefully acknowledge research support from these agencies as well as by the Council of Scientific and Industrial Research of India, the Department of Science and Technology, India, the Science \& Engineering Research Board (SERB), India, the Ministry of Human Resource Development, India, the Spanish Agencia Estatal de Investigación, the Vicepresidència i Conselleria d'Innovació, Recercai Turisme and the Conselleria d'Educació i Universitat del Govern de les Illes Balears, the Conselleria d'Innovació, Universitats, Ciència i Societat Digital de la Generalitat Valenciana and the CERCA Programme Generalitat de Catalunya, Spain, the National Science Centre of Poland and the Foundation for Polish Science (FNP), the Swiss National Science Foundation (SNSF), the Russian Foundation for Basic Research, the Russian Science Foundation, the European Commission, the European Regional Development Funds (ERDF), the Royal Society, the Scottish Funding Council, the Scottish Universities Physics Alliance, the Hungarian Scientific Research Fund (OTKA), the French Lyon Institute of Origins (LIO), the Belgian Fonds de la Recherche Scientifique (FRS-FNRS), Actions de Recherche Concertées (ARC) and Fonds Wetenschappelijk OnderzoekVlaanderen (FWO), Belgium, the Paris Île-de-France Region, the National Research, Development and Innovation Office Hungary (NKFIH), the National Research Foundation of Korea, the Natural Science and Engineering Research Council Canada, Canadian Foundation for Innovation (CFI), the Brazilian Ministry of Science, Technology, and Innovations, the International Center for Theoretical Physics South American Institute for Fundamental Research (ICTPSAIFR), the Research Grants Council of Hong Kong, the National Natural Science Foundation of China (NSFC), the Leverhulme Trust, the Research Corporation, the Ministry of Science and Technology (MOST), Taiwan, the United States Department of Energy, and the Kavli Foundation. The authors gratefully acknowledge the support of the NSF, STFC, INFN and CNRS for provision of computational resources. This work was supported by MEXT, JSPS Leading-edge Research Infrastructure Program, JSPS Grant-in-Aid for Specially Promoted Research 26000005(Kajita 2014-2018), JSPS Grant-in-Aid for Scientific Research on Innovative Areas 2905: Grants No. JP17H06358, No. JP17H06361, and No. JP17H06364, JSPS Core-to-Core Program A. Advanced Research Networks, JSPS Grant-in-Aid for Scientific Research (S) Grants No. $17 \mathrm{H} 06133$ and No. 20H05639, JSPS Grant-in-Aid for Transformative Research Areas (A) 20A203: Grant No. JP20H05854, the joint research program of the Institute for Cosmic Ray Research, University of Tokyo, National Research Foundation (NRF) and Computing Infrastructure Project of KISTI-GSDC in Korea, Academia Sinica (AS), AS Grid Center (ASGC) and the Ministry of Science and Technology (MoST) in Taiwan under grants including Grant No. AS-CDA-105-M06, Advanced Technology Center (ATC) of NAOJ, Mechanical Engineering Center of KEK. 
[1] J. Aasi et al. (LIGO Scientific Collaboration), Classical Quantum Gravity 32, 074001 (2015).

[2] F. Acernese et al. (Virgo Collaboration), Classical Quantum Gravity 32, 024001 (2015).

[3] R. Abbott et al. (LIGO Scientific Collaboration and Virgo Collaboration), Phys. Rev. X 11, 021053 (2021).

[4] R. Abbott et al. (LIGO Scientific Collaboration, Virgo Collaboration and KAGRA Collaboration), arXiv:2105.15120.

[5] R. Abbott et al. (LIGO Scientific Collaboration and Virgo Collaboration), Astrophys. J. Lett. 915, L5 (2021).

[6] A. H. Nitz, C. D. Capano, S. Kumar, Y.-F. Wang, S. Kastha, M. Schäfer, R. Dhurkunde, and M. Cabero, arXiv:2105.09151.

[7] B. P. Abbott et al. (LIGO Scientific Collaboration and Virgo Collaboration), Phys. Rev. D 101, 084002 (2020).

[8] B. P. Abbott et al. (LIGO Scientific Collaboration and Virgo Collaboration), Astrophys. J. 874, 163 (2019).

[9] J. Abadie et al. (LIGO Scientific Collaboration), Phys. Rev. D 83, 042001 (2011).

[10] M. Ebersold and S. Tiwari, Phys. Rev. D 101, 104041 (2020).

[11] R. Abbott et al. (LIGO Scientific Collaboration, Virgo Collaboration and KAGRA Collaboration), Phys. Rev. Lett. 126, 241102 (2021).

[12] R. Abbott et al. (LIGO Scientific Collaboration and Virgo Collaboration), arXiv:2105.06384.

[13] B. P. Abbott et al. (LIGO Scientific Collaboration, Virgo Collaboration), Phys. Rev. D 100, 024017 (2019).

[14] B. P. Abbott et al. (LIGO Scientific Collaboration and Virgo Collaboration), Phys. Rev. D 99, 104033 (2019).

[15] B. P. Abbott et al. (LIGO Scientific Collaboration and Virgo Collaboration), Phys. Rev. D 95, 042003 (2017).

[16] L. Sun et al., Classical Quantum Gravity 37, 225008 (2020).

[17] L. Sun, J. Kissel, E. Goetz, and J. Betzweiser, Characterization of systematic error in Advanced LIGO calibration in the second half of $\mathrm{O} 3$ (O3B), LIGO Technical Report No. LIGO-T2000584, 2021.

[18] D. Estevez, B. Mours, L. Rolland, and D.Verkindt, Online $\mathrm{h}(\mathrm{t})$ reconstruction for Virgo O3 data: start of O3, Virgo Technical Report No. VIR-0652B-19, 2019.

[19] L. Rolland, D. Estevez, P. Lagabbe, B. Mours, M. SeglarArroyo, T. Pradier, and V. D., Update on h(t) uncertainties during O3, Virgo Technical Report No. VIR-0688A-20, 2020.

[20] D. Davis et al., Classical Quantum Gravity 38, 135014 (2021).

[21] https://emfollow.docs.ligo.org/userguide/.

[22] R. Abbott et al. (LIGO Scientific Collaboration and Virgo Collaboration), SoftwareX 13, 100658 (2021).

[23] LIGO Scientific Collaboration And Virgo Collaboration, LIGO Virgo strain data from observing run O3a, https:// www.gw-openscience.org/O3/O3a (2021).

[24] A. Buikema et al., Phys. Rev. D 102, 062003 (2020).

[25] B. P. Abbott et al., Classical Quantum Gravity 33, 134001 (2016).

[26] P. Nguyen et al., Classical Quantum Gravity 38, 145001 (2021).

[27] A. Effler, R. M. S. Schofield, V. V. Frolov, G. González, K. Kawabe, J. R. Smith, J. Birch, and R. McCarthy, Classical Quantum Gravity 32, 035017 (2015).

[28] B. P. Abbott et al. (LIGO Scientific Collaboration and Virgo Collaboration), Classical Quantum Gravity 35, 065010 (2018).
[29] J. R. Smith, T. Abbott, E. Hirose, N. Leroy, D. MacLeod, J. McIver, P. Saulson, and P. Shawhan, Classical Quantum Gravity 28, 235005 (2011).

[30] D. Davis, B. Hughey, T. Massinger, L. Nuttall, A. Stuver, and J. Zweizig, Data quality vetoes applied to the analysis of LIGO data from the third observing run, LVK Technical Report No. LIGO-T2100045, 2021.

[31] S. Soni et al., Classical Quantum Gravity 38, 025016 (2021).

[32] S. Soni et al., arXiv:2103.12104.

[33] M. Cabero, A. Lundgren, A. H. Nitz, T. Dent, D. Barker, E. Goetz, J. S. Kissel, L. K. Nuttall, P. Schale, R. Schofield et al., Classical Quantum Gravity 36, 155010 (2019).

[34] S. Klimenko, G. Vedovato, M. Drago, F. Salemi, V. Tiwari, G. A. Prodi, C. Lazzaro, S. Tiwari, F. Da Silva, and G. Mitselmakher, Phys. Rev. D 93, 042004 (2016).

[35] M. Drago et al., SoftwareX 14, 100678 (2021).

[36] https://gwburst.gitlab.io.

[37] V. Necula, S. Klimenko, and G. Mitselmakher, J. Phys. Conf. Ser. 363, 012032 (2012).

[38] N. Cornish and T. Littenberg, Classical Quantum Gravity 32, 135012 (2015).

[39] https://git.ligo.org/lscsoft/bayeswave.

[40] N. J. Cornish, T. B. Littenberg, B. Bécsy, K. Chatziioannou, J. A. Clark, S. Ghonge, and M. Millhouse, Phys. Rev. D 103, 044006 (2021).

[41] LIGO Scientific Collaboration and Virgo Collaboration, GCN 25883 (2019), https://gcn.gsfc.nasa.gov/other/GW $190928 \mathrm{c} \cdot g \mathrm{cn} 3$.

[42] M. Zevin, S. Coughlin, S. Bahaadini, E. Besler, N. Rohani, S. Allen, M. Cabero, K. Crowston, A. Katsaggelos, S. Larson, T. Lee, C. Lintott, T. Littenberg, A. Lundgren, C. Osterlund, J. Smith, L. Trouille, and V. Kalogera, Classical Quantum Gravity 34, 064003 (2017).

[43] LIGO Scientific Collaboration and Virgo Collaboration, GCN 26220 (2019), https://gcn.gsfc.nasa.gov/other/ GW191110af.gcn3.

[44] LIGO Scientific Collaboration and Virgo Collaboration, GCN 26731 (2020), https://gcn.gsfc.nasa.gov/other/GW 200114f.gen3.

[45] J. Abadie et al. (LIGO Scientific Collaboration and Virgo Collaboration), Phys. Rev. D 85, 122007 (2012).

[46] J. B. Kanner, T. B. Littenberg, N. Cornish, M. Millhouse, E. Xhakaj, F. Salemi, M. Drago, G. Vedovato, and S. Klimenko, Phys. Rev. D 93, 022002 (2016).

[47] Y. S. C. Lee, M. Millhouse, and A. Melatos, Phys. Rev. D 103, 062002 (2021).

[48] P. J. Sutton, arXiv:1304.0210.

[49] E. Abdikamalov, G. Pagliaroli, and D. Radice, arXiv:2010 .04356 .

[50] B. P. Abbott et al., Phys. Rev. D 94, 102001 (2016).

[51] E. O'Connor, The core-collapse supernova-black hole connection, in Handbook of Supernovae, edited by A.W. Alsabti and P. Murdin (Springer publishing, New York, 2017), p. 1555.

[52] M. J. Szczepańczyk et al., arXiv:2104.06462 [Phys. Rev. D (to be published)].

[53] J. Powell and B. Müller, Mon. Not. R. Astron. Soc. 487, 1178 (2019).

[54] E. P. O’Connor and S. M. Couch, Astrophys. J. 865, 81 (2018). 
[55] J. M. Blondin, A. Mezzacappa, and C. DeMarino, Astrophys. J. 584, 971 (2003).

[56] J. M. Blondin and A. Mezzacappa, Astrophys. J. 642, 401 (2006).

[57] T. Foglizzo, P. Galletti, L. Scheck, and H.-T. Janka, Astrophys. J. 654, 1006 (2007).

[58] D. Radice, V. Morozova, A. Burrows, D. Vartanyan, and H. Nagakura, Astrophys. J. Lett. 876, L9 (2019).

[59] H.-T. Janka, Annu. Rev. Nucl. Part. Sci. 62, 407 (2012).

[60] S. Woosley and A. Heger, Astrophys. J. 637, 914 (2006).

[61] J. Powell and B. Müller, Mon. Not. R. Astron. Soc. 494, 4665 (2020).

[62] M. Obergaulinger and M. Á. Aloy, Mon. Not. R. Astron. Soc. 492, 4613 (2020).

[63] K. Abe, Y. Haga, Y. Hayato, M. Ikeda, K. Iyogi, J. Kameda, Y. Kishimoto, M. Miura, S. Moriyama, M. Nakahata, Y. Nakano, S. Nakayama, H. Sekiya, M. Shiozawa, Suzuki et al., Astropart. Phys. 81, 39 (2016).

[64] P. J. McMillan, Mon. Not. R. Astron. Soc. 465, 76 (2017).

[65] M. Cautun, A. Benítez-Llambay, A. J. Deason, C. S. Frenk, A. Fattahi, F. A. Gómez, R. J. J. Grand, K. A. Oman, J. F. Navarro, and C. M. Simpson, Mon. Not. R. Astron. Soc. 494, 4291 (2020).

[66] B. P. Abbott et al. (LIGO Scientific Collaboration and Virgo Collaboration), Astrophys. J. 874, 163 (2019).

[67] B. Haskell and A. Melatos, Int. J. Mod. Phys. D 24, 1530008 (2015).

[68] N. Andersson, G. L. Comer, and R. Prix, Mon. Not. R. Astron. Soc. 354, 101 (2004).
[69] L. Warszawski and A. Melatos, Mon. Not. R. Astron. Soc. 423, 2058 (2012).

[70] W. C. G. Ho, D. I. Jones, N. Andersson, and C. M. Espinoza, Phys. Rev. D 101, 103009 (2020).

[71] P. Caraveo, A. Luca, and R. Mignani, Astrophys. J. 561, 930 (2001).

[72] R. N. Manchester, G. B. Hobbs, A. Teoh, and M. Hobbs, VizieR Online Data Catalog, VII/245 (2005).

[73] D. D. Doneva, E. Gaertig, K. D. Kokkotas, and C. Krüger, Phys. Rev. D 88, 044052 (2013).

[74] A. Akmal, V. R. Pandharipande, and D. G. Ravenhall, Phys. Rev. C 58, 1804 (1998).

[75] B. D. Lackey, M. Nayyar, and B. J. Owen, Phys. Rev. D 73, 024021 (2006).

[76] B. P. Abbott et al. (LIGO Scientific Collaboration and Virgo Collaboration), Phys. Rev. Lett. 121, 161101 (2018).

[77] B. P. Abbott et al. (LIGO Scientific Collaboration and Virgo Collaboration), Classical Quantum Gravity 37, 045006 (2020).

[78] J. R. Fuentes, C. M. Espinoza, A. Reisenegger, B. Shaw, B. W. Stappers, and A. G. Lyne, Astron. Astrophys. 608, A131 (2017).

[79] M. Yu, R. N. Manchester, G. Hobbs, S. Johnston, V. M. Kaspi, M. Keith, A. G. Lyne, G. J. Qiao, V. Ravi, J. M. Sarkissian, R. Shannon, and R. X. Xu, Mon. Not. R. Astron. Soc. 429, 688 (2013).

[80] C. M. Espinoza, A. G. Lyne, B. W. Stappers, and M. Kramer, Mon. Not. R. Astron. Soc. 414, 1679 (2011).

[81] B. P. Abbott et al. (LIGO Scientific Collaboration and Virgo Collaboration), Living Rev. Relativity 23, 3 (2020).

R. Abbott,,${ }^{1}$ T. D. Abbott, ${ }^{2}$ F. Acernese,${ }^{3,4}$ K. Ackley, ${ }^{5}$ C. Adams,${ }^{6}$ N. Adhikari, ${ }^{7}$ R. X. Adhikari, ${ }^{1}$ V. B. Adya,${ }^{8}$ C. Affeldt,${ }^{9,10}$ D. Agarwal, ${ }^{11}$ M. Agathos, ${ }^{12,13}$ K. Agatsuma, ${ }^{14}$ N. Aggarwal,${ }^{15}$ O. D. Aguiar, ${ }^{16}$ L. Aiello, ${ }^{17}$ A. Ain, ${ }^{18}$ P. Ajith, ${ }^{19}$ T. Akutsu, ${ }^{20,21}$ S. Albanesi, ${ }^{22}$ A. Allocca, ${ }^{23,4}$ P. A. Altin, ${ }^{8}$ A. Amato, ${ }^{24}$ C. Anand, ${ }^{5}$ S. Anand, ${ }^{1}$ A. Ananyeva, ${ }^{1}$ S. B. Anderson, ${ }^{1}$ W. G. Anderson, ${ }^{7}$ M. Ando,${ }^{25,26}$ T. Andrade, ${ }^{27}$ N. Andres, ${ }^{28}$ T. Andrić, ${ }^{29}$ S. V. Angelova, ${ }^{30}$ S. Ansoldi,${ }^{31,32}$ J. M. Antelis, ${ }^{33}$ S. Antier, ${ }^{34}$ S. Appert, ${ }^{1}$ Koji Arai, ${ }^{1}$ Koya Arai,${ }^{35}$ Y. Arai, ${ }^{35}$ S. Araki, ${ }^{36}$ A. Araya,${ }^{37}$ M. C. Araya, ${ }^{1}$ J. S. Areeda, ${ }^{38}$ M. Arène,${ }^{34}$ N. Aritomi, ${ }^{25}$ N. Arnaud,${ }^{39,40}$ S. M. Aronson, ${ }^{2}$ K. G. Arun, ${ }^{41}$ H. Asada, ${ }^{42}$ Y. Asali, ${ }^{43}$ G. Ashton, ${ }^{5}$ Y. Aso, ${ }^{44,45}$ M. Assiduo, ${ }^{46,47}$ S. M. Aston, ${ }^{6}$ P. Astone,${ }^{48}$ F. Aubin, ${ }^{28}$ C. Austin, ${ }^{2}$ S. Babak, ${ }^{34}$ F. Badaracco, ${ }^{49}$

M. K. M. Bader ${ }^{50}$ C. Badger, ${ }^{51}$ S. Bae,${ }^{52}$ Y. Bae,${ }^{53}$ A. M. Baer,${ }^{54}$ S. Bagnasco, ${ }^{22}$ Y. Bai, ${ }^{1}$ L. Baiotti,${ }^{55}$ J. Baird, ${ }^{34}$ R. Bajpai, ${ }^{56}$ M. Ball ${ }^{57}$ G. Ballardin ${ }^{40}$ S. W. Ballmer, ${ }^{58}$ A. Balsamo, ${ }^{54}$ G. Baltus, ${ }^{59}$ S. Banagiri, ${ }^{60}$ D. Bankar, ${ }^{11}$ J. C. Barayoga, ${ }^{1}$ C. Barbieri, ${ }^{61,62,63}$ B. C. Barish, ${ }^{1}$ D. Barker, ${ }^{64}$ P. Barneo, ${ }^{27}$ F. Barone,${ }^{65,4}$ B. Barr, ${ }^{66}$ L. Barsotti, ${ }^{67}$ M. Barsuglia, ${ }^{34}$ D. Barta, ${ }^{68}$ J. Bartlett, ${ }^{64}$ M. A. Barton, ${ }^{66,20}$ I. Bartos, ${ }^{69}$ R. Bassiri, ${ }^{70}$ A. Basti, ${ }^{71,18}$ M. Bawaj, ${ }^{72,73}$ J. C. Bayley, ${ }^{66}$ A. C. Baylor, ${ }^{7}$ M. Bazzan, ${ }^{74,75}$ B. Bécsy, ${ }^{76}$ V. M. Bedakihale, ${ }^{77}$ M. Bejger, ${ }^{78}$ I. Belahcene, ${ }^{39}$ V. Benedetto, ${ }^{79}$ D. Beniwal,${ }^{80}$ T. F. Bennett,${ }^{81}$ J. D. Bentley,${ }^{14}$ M. BenYaala, ${ }^{30}$ F. Bergamin,${ }^{9,10}$ B. K. Berger ${ }^{70}$ S. Bernuzzi, ${ }^{13}$ C. P. L. Berry, ${ }^{15,66}$ D. Bersanetti, ${ }^{82}$ A. Bertolini ${ }^{50}$ J. Betzwieser, ${ }^{6}$ D. Beveridge,${ }^{83}$ R. Bhandare, ${ }^{84}$ U. Bhardwaj, ${ }^{85,50}$ D. Bhattacharjee ${ }^{86}$ S. Bhaumik, ${ }^{69}$ I. A. Bilenko, ${ }^{87}$ G. Billingsley, ${ }^{1}$ S. Bini,${ }^{88,89}$ R. Birney, ${ }^{90}$ O. Birnholtz, ${ }^{91}$ S. Biscans, ${ }^{1,67}$ M. Bischi, ${ }^{46,47}$ S. Biscoveanu, ${ }^{67}$ A. Bisht, ${ }^{9,10}$ B. Biswas, ${ }^{11}$ M. Bitossi, ${ }^{40,18}$ M.-A. Bizouard, ${ }^{92}$ J. K. Blackburn, ${ }^{1}$ C. D. Blair ${ }^{83,6}$ D. G. Blair ${ }^{83}$ R. M. Blair, ${ }^{64}$ F. Bobba,${ }^{93,94}$ N. Bode, ${ }^{9,10}$ M. Boer, ${ }^{92}$ G. Bogaert, ${ }^{92}$ M. Boldrini, ${ }^{95,48}$ L. D. Bonavena, ${ }^{74}$ F. Bondu,${ }^{96}$ E. Bonilla, ${ }^{70}$ R. Bonnand, ${ }^{28}$ P. Booker, ${ }^{910}$ B. A. Boom,${ }^{50}$ R. Bork, ${ }^{1}$ V. Boschi, ${ }^{18}$ N. Bose,${ }^{97}$ S. Bose, ${ }^{11}$ V. Bossilkov, ${ }^{83}$ V. Boudart, ${ }^{59}$ Y. Bouffanais, ${ }^{74,75}$ A. Bozzi, ${ }^{40}$ C. Bradaschia, ${ }^{18}$ P. R. Brady, ${ }^{7}$ A. Bramley, ${ }^{6}$ A. Branch,${ }^{6}$ M. Branchesi, ${ }^{29,98}$ J. E. Brau, ${ }^{57}$ M. Breschi, ${ }^{13}$ T. Briant, ${ }^{99}$ J. H. Briggs, ${ }^{66}$ A. Brillet, ${ }^{92}$ M. Brinkmann, ${ }^{9,10}$ P. Brockill, ${ }^{7}$ A. F. Brooks, ${ }^{1}$ J. Brooks, ${ }^{40}$ D. D. Brown, ${ }^{80}$ S. Brunett, ${ }^{1}$ G. Bruno, ${ }^{49}$ R. Bruntz,${ }^{54}$ J. Bryant, ${ }^{14}$ T. Bulik, ${ }^{100}$ H. J. Bulten, ${ }^{50}$ A. Buonanno, ${ }^{101,102}$ 
R. Buscicchio, ${ }^{14}$ D. Buskulic, ${ }^{28}$ C. Buy, ${ }^{103}$ R. L. Byer, ${ }^{70}$ L. Cadonati,${ }^{104}$ G. Cagnoli, ${ }^{24}$ C. Cahillane,${ }^{64}$ J. Calderón Bustillo, ${ }^{105,106}$ J. D. Callaghan ${ }^{66}$ T. A. Callister, ${ }^{107,108}$ E. Calloni, ${ }^{23,4}$ J. Cameron, ${ }^{83}$ J. B. Camp, ${ }^{109}$ M. Canepa,${ }^{110,82}$ S. Canevarolo, ${ }^{111}$ M. Cannavacciuolo, ${ }^{93}$ K. C. Cannon, ${ }^{112}$ H. Cao,${ }^{80}$ Z. Cao, ${ }^{113}$ E. Capocasa, ${ }^{20}$ E. Capote,${ }^{58}$ G. Carapella, ${ }^{93,94}$ F. Carbognani, ${ }^{40}$ J. B. Carlin, ${ }^{114}$ M. F. Carney, ${ }^{15}$ M. Carpinelli, ${ }^{115,116,40}$ G. Carrillo, ${ }^{57}$ G. Carullo, ${ }^{71,18}$ T. L. Carver, ${ }^{17}$ J. Casanueva Diaz, ${ }^{40}$ C. Casentini, ${ }^{117,118}$ G. Castaldi, ${ }^{119}$ S. Caudill, ${ }^{50,111}$ M. Cavaglià, ${ }^{86}$ F. Cavalier, ${ }^{39}$ R. Cavalieri, ${ }^{40}$ M. Ceasar, ${ }^{120}$ G. Cella, ${ }^{18}$ P. Cerdá-Durán, ${ }^{121}$ E. Cesarini, ${ }^{118}$ W. Chaibi, ${ }^{92}$ K. Chakravarti, ${ }^{11}$ S. Chalathadka Subrahmanya, ${ }^{122}$ E. Champion, ${ }^{123}$ C.-H. Chan, ${ }^{124}$ C. Chan, ${ }^{112}$ C. L. Chan, ${ }^{106}$ K. Chan, ${ }^{106}$ M. Chan, ${ }^{125}$ K. Chandra ${ }^{97}$ P. Chanial, ${ }^{40}$ S. Chao, ${ }^{124}$ P. Charlton, ${ }^{126}$ E. A. Chase,${ }^{15}$ E. Chassande-Mottin, ${ }^{34}$ C. Chatterjee,${ }^{83}$ Debarati Chatterjee, ${ }^{11}$ Deep Chatterjee, ${ }^{7}$ M. Chaturvedi, ${ }^{84}$ S. Chaty, ${ }^{34}$ K. Chatziioannou, ${ }^{1}$ C. Chen, ${ }^{127,128}$ H. Y. Chen, ${ }^{67}$ J. Chen, ${ }^{124}$ K. Chen, ${ }^{129}$ X. Chen, ${ }^{83}$ Y.-B. Chen, ${ }^{130}$ Y.-R. Chen, ${ }^{131}$ Z. Chen, ${ }^{17}$ H. Cheng, ${ }^{69}$ C. K. Cheong, ${ }^{106}$ H. Y. Cheung, ${ }^{106}$ H. Y. Chia, ${ }^{69}$ F. Chiadini, ${ }^{132,94}$ C-Y. Chiang, ${ }^{133}$ G. Chiarini, ${ }^{75}$ R. Chierici, ${ }^{134}$ A. Chincarini, ${ }^{82}$ M. L. Chiofalo, ${ }^{71,18}$ A. Chiummo,${ }^{40}$ G. Cho, ${ }^{135}$ H. S. Cho, ${ }^{136}$ R. K. Choudhary ${ }^{83}$ S. Choudhary ${ }^{11}$ N. Christensen, ${ }^{92}$ H. Chu, ${ }^{129}$ Q. Chu, ${ }^{83}$ Y-K. Chu, ${ }^{133}$ S. Chua, ${ }^{8}$ K. W. Chung, ${ }^{51}$ G. Ciani,${ }^{74,75}$ P. Ciecielag, ${ }^{78}$ M. Cieślar, ${ }^{78}$ M. Cifaldi, ${ }^{117,118}$ A. A. Ciobanu, ${ }^{80}$ R. Ciolfi, ${ }^{137,75}$ F. Cipriano, ${ }^{92}$ A. Cirone,${ }^{110,82}$ F. Clara,${ }^{64}$ E. N. Clark, ${ }^{138}$ J. A. Clark, ${ }^{1,104}$ L. Clarke, ${ }^{139}$ P. Clearwater, ${ }^{140}$ S. Clesse, ${ }^{141}$ F. Cleva, ${ }^{92}$ E. Coccia, ${ }^{29,98}$ E. Codazzo, ${ }^{29}$ P.-F. Cohadon, ${ }^{99}$ D. E. Cohen, ${ }^{39}$ L. Cohen, ${ }^{2}$ M. Colleoni, ${ }^{142}$ C. G. Collette, ${ }^{143}$ A. Colombo, ${ }^{61}$ M. Colpi,${ }^{61,62}$ C. M. Compton, ${ }^{64}$ M. Constancio Jr.,${ }^{16}$ L. Conti, ${ }^{75}$ S. J. Cooper, ${ }^{14}$ P. Corban, ${ }^{6}$ T. R. Corbitt, ${ }^{2}$ I. Cordero-Carrión, ${ }^{144}$ S. Corezzi, ${ }^{73,72}$ K. R. Corley, ${ }^{43}$ N. Cornish, ${ }^{76}$ D. Corre, ${ }^{39}$ A. Corsi, ${ }^{145}$ S. Cortese, ${ }^{40}$ C. A. Costa, ${ }^{16}$ R. Cotesta, ${ }^{102}$ M. W. Coughlin, ${ }^{60}$ J.-P. Coulon, ${ }^{92}$ S. T. Countryman, ${ }^{43}$ B. Cousins, ${ }^{146}$ P. Couvares, ${ }^{1}$ D. M. Coward, ${ }^{83}$ M. J. Cowart, ${ }^{6}$ D. C. Coyne, ${ }^{1}$ R. Coyne, ${ }^{147}$ J. D. E. Creighton, ${ }^{7}$ T. D. Creighton, ${ }^{148}$ A. W. Criswell, ${ }^{60}$ M. Croquette, ${ }^{99}$ S. G. Crowder, ${ }^{149}$ J. R. Cudell, ${ }^{59}$ T. J. Cullen, ${ }^{2}$ A. Cumming, ${ }^{66}$ R. Cummings, ${ }^{66}$ L. Cunningham, ${ }^{66}$ E. Cuoco, ${ }^{40,150,18}$ M. Curyło, ${ }^{100}$ P. Dabadie, ${ }^{24}$ T. Dal Canton, ${ }^{39}$ S. Dall'Osso, ${ }^{29}$ G. Dálya, ${ }^{151}$ A. Dana, ${ }^{70}$ L. M. DaneshgaranBajastani, ${ }^{81}$ B. D’Angelo, ${ }^{110,82}$ S. Danilishin, ${ }^{152,50}$ S. D’Antonio, ${ }^{118}$ K. Danzmann,, 10 C. Darsow-Fromm, ${ }^{122}$ A. Dasgupta, ${ }^{77}$ L. E. H. Datrier, ${ }^{66}$ S. Datta, ${ }^{11}$ V. Dattilo, ${ }^{40}$ I. Dave, ${ }^{84}$ M. Davier,${ }^{39}$ G. S. Davies, ${ }^{153}$ D. Davis, ${ }^{1}$ M. C. Davis, ${ }^{120}$ E. J. Daw, ${ }^{154}$ R. Dean,${ }^{120}$ D. DeBra ${ }^{70}$ M. Deenadayalan, ${ }^{11}$ J. Degallaix, ${ }^{155}$ M. De Laurentis,${ }^{23,4}$ S. Deléglise,${ }^{99}$ V. Del Favero, ${ }^{123}$ F. De Lillo, ${ }^{49}$ N. De Lillo, ${ }^{66}$ W. Del Pozzo,${ }^{71,18}$ L. M. DeMarchi, ${ }^{15}$ F. De Matteis, ${ }^{117,118}$ V. D'Emilio, ${ }^{17}$ N. Demos, ${ }^{67}$ T. Dent,${ }^{105}$ A. Depasse, ${ }^{49}$ R. De Pietri, ${ }^{156,157}$ R. De Rosa, ${ }^{23,4}$ C. De Rossi,${ }^{40}$ R. DeSalvo, ${ }^{119}$ R. De Simone, ${ }^{132}$ S. Dhurandhar, ${ }^{11}$ M. C. Díaz, ${ }^{148}$ M. Diaz-Ortiz Jr., ${ }^{69}$ N. A. Didio ${ }^{58}$ T. Dietrich, ${ }^{102,50}$ L. Di Fiore, ${ }^{4}$ C. Di Fronzo, ${ }^{14}$ C. Di Giorgio, ${ }^{93,94}$ F. Di Giovanni, ${ }^{121}$ M. Di Giovanni ${ }^{29}$ T. Di Girolamo, ${ }^{23,4}$ A. Di Lieto, ${ }^{71,18}$ B. Ding, ${ }^{143}$ S. Di Pace,${ }^{95,48}$ I. Di Palma, ${ }^{95,48}$ F. Di Renzo, ${ }^{71,18}$ A. K. Divakarla, ${ }^{69}$ A. Dmitriev, ${ }^{14}$ Z. Doctor, ${ }^{57}$ L. D'Onofrio, ${ }^{23,4}$ F. Donovan, ${ }^{67}$ K. L. Dooley, ${ }^{17}$ S. Doravari, ${ }^{11}$ I. Dorrington, ${ }^{17}$ M. Drago, ${ }^{95,48}$ J. C. Driggers,${ }^{64}$ Y. Drori, ${ }^{1}$ J.-G. Ducoin,${ }^{39}$ P. Dupej, ${ }^{66}$ O. Durante, ${ }^{93,94}$ D. D’Urso, ${ }^{115,116}$ P.-A. Duverne, ${ }^{39}$ S. E. Dwyer, ${ }^{64}$ C. Eassa, ${ }^{64}$ P. J. Easter, ${ }^{5}$ M. Ebersold, ${ }^{158}$ T. Eckhardt, ${ }^{122}$ G. Eddolls, ${ }^{66}$ B. Edelman,${ }^{57}$ T. B. Edo, ${ }^{1}$ O. Edy ${ }^{153}$ A. Effler, ${ }^{6}$ S. Eguchi, ${ }^{125}$ J. Eichholz, ${ }^{8}$ S. S. Eikenberry, ${ }^{69}$ M. Eisenmann, ${ }^{28}$ R. A. Eisenstein, ${ }^{67}$ A. Ejlli, ${ }^{17}$ E. Engelby, ${ }^{38}$ Y. Enomoto ${ }^{25}$ L. Errico, ${ }^{23,4}$ R. C. Essick, ${ }^{159}$ H. Estellés, ${ }^{142}$ D. Estevez, ${ }^{160}$ Z. Etienne, ${ }^{161}$ T. Etzel, ${ }^{1}$ M. Evans, ${ }^{67}$ T. M. Evans, ${ }^{6}$ B. E. Ewing, ${ }^{146}$ V. Fafone, ${ }^{117,118,29}$ H. Fair, ${ }^{58}$ S. Fairhurst,${ }^{17}$ A. M. Farah, ${ }^{159}$ S. Farinon, ${ }^{82}$ B. Farr, ${ }^{57}$ W. M. Farr, ${ }^{107,108}$ N. W. Farrow, ${ }^{5}$ E. J. Fauchon-Jones, ${ }^{17}$ G. Favaro,${ }^{74}$ M. Favata, ${ }^{162}$ M. Fays ${ }^{59}$ M. Fazio, ${ }^{163}$ J. Feicht, ${ }^{1}$ M. M. Fejer, ${ }^{70}$ E. Fenyvesi, ${ }^{68,164}$ D. L. Ferguson, ${ }^{165}$ A. Fernandez-Galiana, ${ }^{67}$ I. Ferrante, ${ }^{71,18}$ T. A. Ferreira, ${ }^{16}$ F. Fidecaro, ${ }^{71,18}$ P. Figura, ${ }^{100}$ I. Fiori, ${ }^{40}$ M. Fishbach, ${ }^{15}$ R. P. Fisher, ${ }^{54}$ R. Fittipaldi, ${ }^{166,94}$ V. Fiumara ${ }^{167,94}$ R. Flaminio, ${ }^{28,20}$ E. Floden ${ }^{60}$ H. Fong, ${ }^{112}$ J. A. Font, ${ }^{121,168}$ B. Fornal, ${ }^{169}$ P. W. F. Forsyth, ${ }^{8}$ A. Franke, ${ }^{122}$ S. Frasca, ${ }^{95,48}$ F. Frasconi, ${ }^{18}$ C. Frederick, ${ }^{170}$ J. P. Freed, ${ }^{33}$ Z. Frei, ${ }^{151}$ A. Freise,${ }^{171}$ R. Frey,${ }^{57}$ P. Fritschel, ${ }^{67}$ V. V. Frolov, ${ }^{6}$ G. G. Fronzé, ${ }^{22}$ Y. Fujii, ${ }^{172}$ Y. Fujikawa, ${ }^{173}$ M. Fukunaga,${ }^{35}$ M. Fukushima, ${ }^{21}$ P. Fulda, ${ }^{69}$ M. Fyffe, ${ }^{6}$ H. A. Gabbard, ${ }^{66}$

B. U. Gadre, ${ }^{102}$ J. R. Gair, ${ }^{102}$ J. Gais, ${ }^{106}$ S. Galaudage, ${ }^{5}$ R. Gamba, ${ }^{13}$ D. Ganapathy, ${ }^{67}$ A. Ganguly, ${ }^{19}$ D. Gao, ${ }^{174}$ S. G. Gaonkar, ${ }^{11}$ B. Garaventa, ${ }^{82,110}$ C. García-Núñez, ${ }^{90}$ C. García-Quirós, ${ }^{142}$ F. Garufi, ${ }^{23,4}$ B. Gateley, ${ }^{64}$ S. Gaudio, ${ }^{33}$ V. Gayathri, ${ }^{69}$ G.-G. Ge, ${ }^{174}$ G. Gemme, ${ }^{82}$ A. Gennai, ${ }^{18}$ J. George, ${ }^{84}$ O. Gerberding, ${ }^{122}$ L. Gergely, ${ }^{175}$ P. Gewecke, ${ }^{122}$ S. Ghonge, ${ }^{104}$ Abhirup Ghosh, ${ }^{102}$ Archisman Ghosh ${ }^{176}$ Shaon Ghosh, ${ }^{7,162}$ Shrobana Ghosh ${ }^{17}$ B. Giacomazzo, ${ }^{61,62,63}$ L. Giacoppo, ${ }^{95,48}$ J. A. Giaime, ${ }^{2,6}$ K. D. Giardina, ${ }^{6}$ D. R. Gibson, ${ }^{90}$ C. Gier,${ }^{30}$ M. Giesler, ${ }^{177}$ P. Giri, ${ }^{18,71}$ F. Gissi, ${ }^{79}$ J. Glanzer, ${ }^{2}$ A. E. Gleckl, ${ }^{38}$ P. Godwin, ${ }^{146}$ E. Goetz, ${ }^{178}$ R. Goetz, ${ }^{69}$ N. Gohlke, ${ }^{9,10}$ B. Goncharov, ${ }^{5,29}$ G. González, ${ }^{2}$ A. Gopakumar, ${ }^{179}$ M. Gosselin, ${ }^{40}$ R. Gouaty,${ }^{28}$ D. W. Gould, ${ }^{8}$ B. Grace, ${ }^{8}$ A. Grado, ${ }^{180,4}$ M. Granata, ${ }^{155}$ V. Granata, ${ }^{93}$ A. Grant, ${ }^{66}$ S. Gras, ${ }^{67}$ P. Grassia, ${ }^{1}$ C. Gray,${ }^{64}$ R. Gray, ${ }^{66}$ G. Greco, ${ }^{72}$ A. C. Green, ${ }^{69}$ R. Green, ${ }^{17}$ A. M. Gretarsson, ${ }^{33}$ E. M. Gretarsson, ${ }^{33}$ D. Griffith, ${ }^{1}$ W. Griffiths, ${ }^{17}$ H. L. Griggs, ${ }^{104}$ G. Grignani, ${ }^{73,72}$ A. Grimaldi, ${ }^{88,89}$ S. J. Grimm, ${ }^{29,98}$ 
H. Grote, ${ }^{17}$ S. Grunewald, ${ }^{102}$ P. Gruning, ${ }^{39}$ D. Guerra, ${ }^{121}$ G. M. Guidi, ${ }^{46,47}$ A. R. Guimaraes, ${ }^{2}$ G. Guixé, ${ }^{27}$ H. K. Gulati, ${ }^{77}$ H.-K. Guo, ${ }^{169}$ Y. Guo, ${ }^{50}$ Anchal Gupta, ${ }^{1}$ Anuradha Gupta, ${ }^{181}$ P. Gupta, ${ }^{50,111}$ E. K. Gustafson, ${ }^{1}$ R. Gustafson, ${ }^{182}$ F. Guzman, ${ }^{183}$ S. Ha, ${ }^{184}$ L. Haegel,${ }^{34}$ A. Hagiwara, ${ }^{35,185}$ S. Haino, ${ }^{133}$ O. Halim,,${ }^{32,186}$ E. D. Hall, ${ }^{67}$ E. Z. Hamilton, ${ }^{158}$ G. Hammond, ${ }^{66}$ W.-B. Han,${ }^{187}$ M. Haney, ${ }^{158}$ J. Hanks, ${ }^{64}$ C. Hanna, ${ }^{146}$ M. D. Hannam,${ }^{17}$ O. Hannuksela,${ }^{111,50}$ H. Hansen, ${ }^{64}$ T. J. Hansen, ${ }^{33}$ J. Hanson, ${ }^{6}$ T. Harder, ${ }^{92}$ T. Hardwick, ${ }^{2}$ K. Haris, ${ }^{50,111}$ J. Harms, ${ }^{29,98}$ G. M. Harry, ${ }^{188}$ I. W. Harry, ${ }^{153}$

D. Hartwig, ${ }^{122}$ K. Hasegawa, ${ }^{35}$ B. Haskell, ${ }^{78}$ R. K. Hasskew, ${ }^{6}$ C.-J. Haster,${ }^{67}$ K. Hattori, ${ }^{189}$ K. Haughian, ${ }^{66}$ H. Hayakawa, ${ }^{190}$ K. Hayama, ${ }^{125}$ F. J. Hayes, ${ }^{66}$ J. Healy, ${ }^{123}$ A. Heidmann, ${ }^{99}$ A. Heidt,${ }^{9,10}$ M. C. Heintze, ${ }^{6}$ J. Heinze, ${ }^{9,10}$ J. Heinzel, ${ }^{191}$ H. Heitmann, ${ }^{92}$ F. Hellman, ${ }^{192}$ P. Hello ${ }^{39}$ A. F. Helmling-Cornell, ${ }^{57}$ G. Hemming, ${ }^{40}$ M. Hendry, ${ }^{66}$ I. S. Heng,${ }^{66}$ E. Hennes,${ }^{50}$ J. Hennig, ${ }^{193}$ M. H. Hennig, ${ }^{193}$ A. G. Hernandez, ${ }^{81}$ F. Hernandez Vivanco, ${ }^{5}$ M. Heurs, ${ }^{9,10}$ S. Hild, ${ }^{152,50}$ P. Hill, ${ }^{30}$ Y. Himemoto, ${ }^{194}$ A. S. Hines, ${ }^{183}$ Y. Hiranuma, ${ }^{195}$ N. Hirata, ${ }^{20}$ E. Hirose, ${ }^{35}$ S. Hochheim,,${ }^{910}$ D. Hofman, ${ }^{155}$ J. N. Hohmann, ${ }^{122}$ D. G. Holcomb, ${ }^{120}$ N. A. Holland, ${ }^{8}$ I. J. Hollows, ${ }^{154}$ Z. J. Holmes, ${ }^{80}$ K. Holt, ${ }^{6}$ D. E. Holz, ${ }^{159}$ Z. Hong, ${ }^{196}$ P. Hopkins, ${ }^{17}$ J. Hough ${ }^{66}$ S. Hourihane,${ }^{130}$ E. J. Howell, ${ }^{83}$ C. G. Hoy, ${ }^{17}$ D. Hoyland,${ }^{14}$ A. Hreibi, ${ }^{9}$.10 B-H. Hsieh, ${ }^{35}$ Y. Hsu, ${ }^{124}$ G-Z. Huang, ${ }^{196}$ H-Y. Huang, ${ }^{133}$ P. Huang, ${ }^{174}$ Y-C. Huang, ${ }^{131}$ Y.-J. Huang, ${ }^{133}$ Y. Huang, ${ }^{67}$ M. T. Hübner, ${ }^{5}$ A. D. Huddart, ${ }^{139}$ B. Hughey ${ }^{33}$ D. C. Y. Hui, ${ }^{197}$ V. Hui, ${ }^{28}$ S. Husa, ${ }^{142}$ S. H. Huttner,${ }^{66}$ R. Huxford, ${ }^{146}$ T. Huynh-Dinh, ${ }^{6}$ S. Ide, ${ }^{198}$ B. Idzkowski, ${ }^{100}$ A. Iess, ${ }^{117,118}$ B. Ikenoue, ${ }^{21} \mathrm{~S}$. Imam, ${ }^{196} \mathrm{~K}$. Inayoshi, ${ }^{199} \mathrm{C}$. Ingram, ${ }^{80} \mathrm{Y}$. Inoue, ${ }^{129} \mathrm{~K}$. Ioka, ${ }^{200} \mathrm{M}$. Isi, ${ }^{67}$ K. Isleif, ${ }^{122}$ K. Ito, ${ }^{201}$ Y. Itoh, ${ }^{202,203}$ B. R. Iyer, ${ }^{19}$ K. Izumi, ${ }^{204}$ V. JaberianHamedan, ${ }^{83}$ T. Jacqmin, ${ }^{99}$ S. J. Jadhav, ${ }^{205}$ S. P. Jadhav, ${ }^{11}$ A. L. James,${ }^{17}$ A. Z. Jan, ${ }^{123}$ K. Jani ${ }^{206}$ J. Janquart, ${ }^{111,50}$ K. Janssens, ${ }^{207,92}$ N. N. Janthalur, ${ }^{205}$ P. Jaranowski, ${ }^{208}$ D. Jariwala, ${ }^{69}$ R. Jaume, ${ }^{142}$ A. C. Jenkins, ${ }^{51}$ K. Jenner, ${ }^{80}$ C. Jeon, ${ }^{209}$ M. Jeunon, ${ }^{60}$ W. Jia,${ }^{67}$ H.-B. Jin, ${ }^{210,211}$ G. R. Johns,${ }^{54}$ A. W. Jones, ${ }^{83}$ D. I. Jones, ${ }^{212}$ J. D. Jones,${ }^{64}$ P. Jones, ${ }^{14}$ R. Jones, ${ }^{66}$ R. J. G. Jonker, ${ }^{50}$ L. Ju, ${ }^{83}$ P. Jung, ${ }^{53}$ K. Jung, ${ }^{184}$ J. Junker, ${ }^{9,10}$ V. Juste, ${ }^{160}$ K. Kaihotsu, ${ }^{201}$ T. Kajita, ${ }^{213}$ M. Kakizaki, ${ }^{189}$ C. V. Kalaghatgi, ${ }^{17,111}$ V. Kalogera, ${ }^{15}$ B. Kamai, ${ }^{1}$ M. Kamiizumi, ${ }_{190}$ N. Kanda, ${ }^{202,203}$ S. Kandhasamy, ${ }^{11}$ G. Kang, ${ }^{214}$ J. B. Kanner, ${ }^{1}$ Y. Kao, ${ }^{124}$ S. J. Kapadia, ${ }^{19}$ D. P. Kapasi, ${ }^{8}$ S. Karat, ${ }^{1}$ C. Karathanasis, ${ }^{215}$ S. Karki ${ }^{86}$ R. Kashyap, ${ }^{146}$ M. Kasprzack, ${ }^{1}$ W. Kastaun, ${ }^{9,10}$ S. Katsanevas, ${ }^{40}$

E. Katsavounidis, ${ }^{67}$ W. Katzman, ${ }^{6}$ T. Kaur,${ }^{83}$ K. Kawabe, ${ }^{64}$ K. Kawaguchi, ${ }^{35}$ N. Kawai, ${ }^{216}$ T. Kawasaki, ${ }^{25}$ F. Kéfélian, ${ }^{92}$ D. Keitel, ${ }^{142}$ J. S. Key, ${ }^{217}$ S. Khadka ${ }^{70}$ F. Y. Khalili, ${ }^{87}$ S. Khan, ${ }^{17}$ E. A. Khazanov, ${ }^{218}$ N. Khetan,${ }^{29,98}$ M. Khursheed, ${ }^{84}$ N. Kijbunchoo, ${ }^{8}$ C. Kim, ${ }^{219}$ J. C. Kim, ${ }^{220}$ J. Kim, ${ }^{221}$ K. Kim, ${ }^{222}$ W. S. Kim, ${ }^{223}$ Y.-M. Kim, ${ }^{224}$ C. Kimball, ${ }^{15}$ N. Kimura, ${ }^{185}$ M. Kinley-Hanlon, ${ }^{66}$ R. Kirchhoff, ${ }^{9,10}$ J. S. Kissel, ${ }^{64}$ N. Kita, ${ }^{25}$ H. Kitazawa, ${ }^{201}$ L. Kleybolte, ${ }^{122}$ S. Klimenko, ${ }^{69}$ A. M. Knee, ${ }^{178}$ T. D. Knowles, ${ }^{161}$ E. Knyazev, ${ }^{67}$ P. Koch,,${ }^{9,10}$ G. Koekoek,${ }^{50,152}$ Y. Kojima, ${ }^{225}$ K. Kokeyama, ${ }^{226}$ S. Koley, ${ }^{29}$ P. Kolitsidou, ${ }^{17}$ M. Kolstein, ${ }^{215}$ K. Komori, ${ }^{67,25}$ V. Kondrashov, ${ }^{1}$ A. K. H. Kong, ${ }^{227}$ A. Kontos, ${ }^{228}$ N. Koper, ${ }^{9,10}$ M. Korobko, ${ }^{122}$ K. Kotake, ${ }^{125}$ M. Kovalam, ${ }^{83}$ D. B. Kozak, ${ }^{1}$ C. Kozakai, ${ }^{44}$ R. Kozu, ${ }^{190}$ V. Kringel, ${ }^{9,10}$ N. V. Krishnendu, ${ }^{9,10}$ A. Królak, ${ }^{229,230}$ G. Kuehn, ${ }^{9,10}$ F. Kuei, ${ }^{124}$ P. Kuijer, ${ }^{50}$ A. Kumar, ${ }^{205}$ P. Kumar, ${ }^{177}$ Rahul Kumar, ${ }^{64}$ Rakesh Kumar, ${ }^{77}$ J. Kume ${ }^{26}$ K. Kuns, ${ }^{67}$ C. Kuo, ${ }^{129}$ H-S. Kuo, ${ }^{196}$ Y. Kuromiya, ${ }^{201}$ S. Kuroyanagi, ${ }^{231,232}$ K. Kusayanagi, ${ }^{216}$ S. Kuwahara, ${ }^{112}$ K. Kwak, ${ }^{184}$ P. Lagabbe, ${ }^{28}$ D. Laghi, ${ }^{71,18}$ E. Lalande ${ }^{233}$ T. L. Lam, ${ }^{106}$ A. Lamberts, ${ }^{92,234}$ M. Landry, ${ }^{64}$ B. B. Lane, ${ }^{67}$ R. N. Lang, ${ }^{67}$ J. Lange, ${ }^{165}$ B. Lantz, ${ }^{70}$ I. La Rosa, ${ }^{28}$ A. Lartaux-Vollard, ${ }^{39}$ P. D. Lasky, ${ }^{5}$ M. Laxen, ${ }^{6}$ A. Lazzarini, ${ }^{1}$ C. Lazzaro, ${ }^{74,75}$ P. Leaci, ${ }^{95,48}$ S. Leavey,,${ }^{9,10}$ Y. K. Lecoeuche, ${ }^{178}$ H. K. Lee,${ }^{235}$ H. M. Lee,${ }^{135}$ H. W. Lee, ${ }^{220}$ J. Lee, ${ }^{135}$ K. Lee, ${ }^{236}$ R. Lee, ${ }^{131}$ J. Lehmann, ${ }^{9,10}$ A. Lemaître, ${ }^{237}$ M. Leonardi, ${ }^{20}$ N. Leroy, ${ }^{39}$ N. Letendre, ${ }^{28}$ C. Levesque, ${ }^{233}$ Y. Levin,${ }^{5}$ J. N. Leviton, ${ }^{182}$ K. Leyde, ${ }^{34}$ A. K. Y. Li, ${ }^{1}$ B. Li, ${ }^{124} \mathrm{~J}^{24 i},{ }^{15}$ K. L. Li, ${ }^{238}$ T. G. F. Li, ${ }^{106}{ }^{6}$ X. Li, ${ }^{130}$ C-Y. Lin, ${ }^{239}$ F-K. Lin, ${ }^{133}$ F-L. Lin, ${ }^{196}$ H. L. Lin ${ }^{129}$ L. C.-C. Lin, ${ }^{184}$ F. Linde,${ }^{240,50}$ S. D. Linker, ${ }^{81}$ J. N. Linley, ${ }^{66}$ T. B. Littenberg, ${ }^{241}$ G. C. Liu, ${ }^{127}$ J. Liu, ${ }^{9,10}$ K. Liu, ${ }^{124}$ X. Liu, ${ }^{7}$ F. Llamas, ${ }^{148}$ M. Llorens-Monteagudo, ${ }^{121}$ R. K. L. Lo, ${ }^{1}$ A. Lockwood, ${ }^{242}$ L. T. London, ${ }^{67}$ A. Longo, ${ }^{243,244}$ D. Lopez, ${ }^{158}$ M. Lopez Portilla, ${ }^{111}$ M. Lorenzini, ${ }^{17,118}$ V. Loriette, ${ }^{245}$ M. Lormand, ${ }^{6}$ G. Losurdo, ${ }^{18}$ T. P. Lott, ${ }^{104}$ J. D. Lough,,${ }^{9,10}$ C. O. Lousto, ${ }^{123}$ G. Lovelace, ${ }^{38}$ J. F. Lucaccioni, ${ }^{170}$ H. Lück, ${ }^{9,10}$ D. Lumaca, ${ }^{117,118}$ A. P. Lundgren, ${ }^{153}$ L.-W. Luo, ${ }^{133}$ J. E. Lynam,${ }^{54}$ R. Macas, ${ }^{153}$ M. MacInnis, ${ }^{67}$ D. M. Macleod, ${ }^{17}$ I. A. O. MacMillan, ${ }^{1}$ A. Macquet, ${ }^{92}$ I. Magaña Hernandez,${ }^{7}$ C. Magazzù ${ }^{18}$ R. M. Magee,${ }^{1}$ R. Maggiore, ${ }^{14}$ M. Magnozzi,${ }^{82,110}$ S. Mahesh, ${ }^{161}$ E. Majorana,${ }^{95,48}$ C. Makarem, ${ }^{1}$ I. Maksimovic,${ }^{245}$ S. Maliakal, ${ }^{1}$ A. Malik, ${ }^{84}$ N. Man, ${ }^{92}$ V. Mandic,${ }^{60}$ V. Mangano,,${ }^{95,48}$ J. L. Mango, ${ }^{246}$ G. L. Mansell, ${ }^{64,67}$ M. Manske, ${ }^{7}$ M. Mantovani, ${ }^{40}$ M. Mapelli, ${ }^{74,75}$ F. Marchesoni, ${ }^{247,72,248}$ M. Marchio, ${ }^{20}$ F. Marion, ${ }^{28}$ Z. Mark,${ }^{130}$ S. Márka, ${ }^{43}$ Z. Márka, ${ }^{43}$ C. Markakis, ${ }^{12}$ A. S. Markosyan, ${ }^{70}$ A. Markowitz, ${ }^{1}$ E. Maros, ${ }^{1}$ A. Marquina, ${ }^{144}$ S. Marsat, ${ }^{34}$ F. Martelli, ${ }^{46,47}$ I. W. Martin, ${ }^{66}$ R. M. Martin, ${ }^{162}$ M. Martinez,${ }^{215}$ V. A. Martinez ${ }^{69}$ V. Martinez, ${ }^{24}$ K. Martinovic, ${ }^{51}$ D. V. Martynov, ${ }^{14}$ E. J. Marx ${ }^{67}$ H. Masalehdan, ${ }^{122}$ K. Mason,${ }^{67}$ E. Massera,${ }^{154}$ A. Masserot, ${ }^{28}$ T. J. Massinger, ${ }^{67}$ M. Masso-Reid, ${ }^{66}$ S. Mastrogiovanni, ${ }^{34}$ A. Matas, ${ }^{102}$ M. Mateu-Lucena, ${ }^{142}$ F. Matichard ${ }^{1,67}$ M. Matiushechkina, ${ }^{9,10}$ N. Mavalvala, ${ }^{67}$ J. J. McCann, ${ }^{83}$ R. McCarthy, ${ }^{64}$ D. E. McClelland, ${ }^{8}$ 
P. K. McClincy, ${ }^{146}$ S. McCormick, ${ }^{6}$ L. McCuller, ${ }^{67}$ G. I. McGhee, ${ }^{66}$ S. C. McGuire, ${ }^{249}$ C. McIsaac, ${ }^{153}$ J. McIver, ${ }^{178}$

T. McRae, ${ }^{8}$ S. T. McWilliams, ${ }^{161}$ D. Meacher, ${ }^{7}$ M. Mehmet, ${ }^{9,10}$ A. K. Mehta, ${ }^{102}$ Q. Meijer, ${ }^{111}$ A. Melatos, ${ }^{114}$ D. A. Melchor, ${ }^{38}$ G. Mendell, ${ }^{64}$ A. Menendez-Vazquez, ${ }^{215}$ C. S. Menoni, ${ }^{163}$ R. A. Mercer, ${ }^{7}$ L. Mereni, ${ }^{155}$ K. Merfeld, ${ }^{57}$ E. L. Merilh, ${ }^{6}$ J. D. Merritt, ${ }^{57}$ M. Merzougui, ${ }^{92}$ S. Meshkov, ${ }^{1, a}$ C. Messenger, ${ }^{66}$ C. Messick, ${ }^{165}$ P. M. Meyers, ${ }^{114}$ F. Meylahn, ${ }^{9,10}$ A. Mhaske, ${ }^{11}$ A. Miani,${ }^{88,89}$ H. Miao, ${ }^{14}$ I. Michaloliakos,${ }^{69}$ C. Michel,${ }^{155}$ Y. Michimura, ${ }^{25}$ H. Middleton, ${ }^{114}$ L. Milano, ${ }^{23}$ A. L. Miller, ${ }^{49}$ A. Miller, ${ }^{81}$ B. Miller,${ }^{85,50}$ M. Millhouse, ${ }^{114}$ J. C. Mills, ${ }^{17}$ E. Milotti, ${ }^{186,32}$ O. Minazzoli, ${ }^{92,250}$ Y. Minenkov, ${ }^{118}$ N. Mio, ${ }^{251}$ Ll. M. Mir, ${ }^{215}$ M. Miravet-Tenés, ${ }^{121}$ C. Mishra, ${ }^{252}$ T. Mishra, ${ }^{69}$ T. Mistry, ${ }^{154}$ S. Mitra, ${ }^{11}$ V. P. Mitrofanov, ${ }^{87}$ G. Mitselmakher,${ }^{69}$ R. Mittleman, ${ }^{67}$ O. Miyakawa, ${ }^{190}$ A. Miyamoto, ${ }^{202}$ Y. Miyazaki,${ }^{25}$ K. Miyo, ${ }^{190}$

S. Miyoki, ${ }^{190}$ Geoffrey Mo, ${ }^{67}$ E. Moguel, ${ }^{170}$ K. Mogushi, ${ }^{86}$ S. R. P. Mohapatra, ${ }^{67}$ S. R. Mohite, ${ }^{7}$ I. Molina, ${ }^{38}$ M. Molina-Ruiz, ${ }^{192}$ M. Mondin, ${ }^{81}$ M. Montani, ${ }^{46,47}$ C. J. Moore, ${ }^{14}$ D. Moraru, ${ }^{64}$ F. Morawski, ${ }^{78}$ A. More, ${ }^{11}$ C. Moreno,${ }^{33}$ G. Moreno, ${ }^{64}$ Y. Mori, ${ }^{201}$ S. Morisaki, ${ }^{7}$ Y. Moriwaki, ${ }^{189}$ B. Mours, ${ }^{160}$ C. M. Mow-Lowry, ${ }^{14,171}$ S. Mozzon, ${ }^{153}$

F. Muciaccia, ${ }^{95,48}$ Arunava Mukherjee, ${ }^{253}$ D. Mukherjee, ${ }^{146}$ Soma Mukherjee, ${ }^{148}$ Subroto Mukherjee ${ }^{77}$

Suvodip Mukherjee, ${ }^{85}$ N. Mukund, ${ }^{9,10}$ A. Mullavey, ${ }^{6}$ J. Munch, ${ }^{80}$ E. A. Muñiz, ${ }^{58}$ P. G. Murray ${ }^{66}$ R. Musenich, ${ }^{82,110}$ J. Muth, ${ }^{33}$ S. Muusse, ${ }^{80}$ S. L. Nadji, ${ }^{9,10}$ K. Nagano, ${ }^{204}$ S. Nagano, ${ }^{254}$ A. Nagar, ${ }^{22,255}$ K. Nakamura, ${ }^{20}$ H. Nakano, ${ }^{256}$ M. Nakano ${ }^{35}$ R. Nakashima, ${ }^{216}$ Y. Nakayama, ${ }^{201}$ V. Napolano, ${ }^{40}$ I. Nardecchia, ${ }^{117,118}$ T. Narikawa, ${ }^{35}$ L. Naticchioni, ${ }^{48}$ B. Nayak, ${ }^{81}$ R. K. Nayak, ${ }^{257}$ R. Negishi, ${ }^{195}$ B. F. Neil, ${ }^{83}$ J. Neilson, ${ }^{79,94}$ G. Nelemans, ${ }^{258}$ T. J. N. Nelson, ${ }^{6}$ M. Nery, ${ }^{9,10}$ P. Neubauer, ${ }^{170}$ A. Neunzert, ${ }^{217}$ K. Y. Ng, ${ }^{67}$ S. W. S. Ng, ${ }^{80}$ C. Nguyen, ${ }^{34}$ P. Nguyen, ${ }^{57}$ T. Nguyen, ${ }^{67}$ L. Nguyen Quynh, ${ }^{259}$ W.-T. Ni, ${ }^{210,174,131}$ S. A. Nichols, ${ }^{2}$ A. Nishizawa, ${ }^{26}$ S. Nissanke, ${ }^{85,50}$ E. Nitoglia, ${ }^{134}$ F. Nocera, ${ }^{40}$ M. Norman, ${ }^{17}$ C. North, ${ }^{17}$

S. Nozaki, ${ }^{189}$ L. K. Nuttall, ${ }^{153}$ J. Oberling, ${ }^{64}$ B. D. O’Brien, ${ }^{69}$ Y. Obuchi, ${ }^{21}$ J. O’Dell, ${ }^{139}$ E. Oelker, ${ }^{66}$ W. Ogaki, ${ }^{35}$ G. Oganesyan, ${ }^{29,98}$ J. J. Oh, ${ }^{223}$ K. Oh, ${ }^{197}$ S. H. Oh, ${ }^{223}$ M. Ohashi, ${ }^{190}$ N. Ohishi, ${ }^{44}$ M. Ohkawa, ${ }^{173}$ F. Ohme, ${ }^{9,10}$ H. Ohta, ${ }^{112}$

M. A. Okada, ${ }^{16}$ Y. Okutani, ${ }^{198}$ K. Okutomi, ${ }^{190}$ C. Olivetto, ${ }^{40}$ K. Oohara,${ }^{195}$ C. Ooi,${ }^{25}$ R. Oram, ${ }^{6}$ B. O'Reilly, ${ }^{6}$

R. G. Ormiston, ${ }^{60}$ N. D. Ormsby, ${ }^{54}$ L. F. Ortega, ${ }^{69}$ R. O’Shaughnessy, ${ }^{123}$ E. O’Shea ${ }^{177}$ S. Oshino, ${ }^{190}$ S. Ossokine, ${ }^{102}$ C. Osthelder, ${ }^{1}$ S. Otabe, ${ }^{216}$ D. J. Ottaway, ${ }^{80}$ H. Overmier, ${ }^{6}$ A. E. Pace, ${ }^{146}$ G. Pagano, ${ }^{71,18}$ M. A. Page, ${ }^{83}$ G. Pagliaroli, ${ }^{29,98}$ A. Pai, ${ }^{97}$ S. A. Pai, ${ }^{84}$ J. R. Palamos, ${ }^{57}$ O. Palashov, ${ }^{218}$ C. Palomba, ${ }^{48}$ H. Pan, ${ }^{124}$ K. Pan, ${ }^{131,227}$ P. K. Panda,${ }^{205}$ H. Pang, ${ }^{129}$ P. T. H. Pang ${ }^{50,111}$ C. Pankow, ${ }^{15}$ F. Pannarale,${ }^{95,48}$ B. C. Pant,${ }^{84}$ F. H. Panther, ${ }^{83}$ F. Paoletti, ${ }^{18}$ A. Paoli, ${ }^{40}$ A. Paolone, ${ }^{48,260}$ A. Parisi, ${ }^{127}$ H. Park, ${ }^{7}$ J. Park, ${ }^{261}$ W. Parker, ${ }^{6,249}$ D. Pascucci, ${ }^{50}$ A. Pasqualetti, ${ }^{40}$ R. Passaquieti, ${ }^{71,18}$ D. Passuello, ${ }^{18}$ M. Patel,${ }^{54}$ M. Pathak,${ }^{80}$ B. Patricelli, ${ }^{40,18}$ A. S. Patron, ${ }^{2}$ S. Patrone,,${ }^{95,48}$ S. Paul, ${ }^{57}$ E. Payne, ${ }^{5}$ M. Pedraza, ${ }^{1}$ M. Pegoraro, ${ }^{75}$

A. Pele, ${ }^{6}$ F. E. Peña Arellano, ${ }^{190}$ S. Penn, ${ }^{262}$ A. Perego,${ }^{88,89}$ A. Pereira, ${ }^{24}$ T. Pereira, ${ }^{263}$ C. J. Perez, ${ }^{64}$ C. Périgois, ${ }^{28}$ C. C. Perkins, ${ }^{69}$ A. Perreca, ${ }^{88,89}$ S. Perriès, ${ }^{134}$ J. Petermann, ${ }^{122}$ D. Petterson, ${ }^{1}$ H. P. Pfeiffer,${ }^{102}$ K. A. Pham, ${ }^{60}$ K. S. Phukon, ${ }^{50,240}$ O. J. Piccinni, ${ }^{48}$ M. Pichot, ${ }^{92}$ M. Piendibene,${ }^{71,18}$ F. Piergiovanni, ${ }^{46,47}$ L. Pierini, ${ }^{95,48}$ V. Pierro, ${ }^{79,94}$ G. Pillant, ${ }^{40}$ M. Pillas, ${ }^{39}$ F. Pilo, ${ }^{18}$ L. Pinard, ${ }^{155}$ I. M. Pinto, ${ }^{79,94,264}$ M. Pinto, ${ }^{40}$ K. Piotrzkowski, ${ }^{49}$ M. Pirello, ${ }^{64}$ M. D. Pitkin, ${ }^{265}$ E. Placidi, ${ }^{95,48}$ L. Planas, ${ }^{142}$ W. Plastino, ${ }^{243,244}$ C. Pluchar,${ }^{138}$ R. Poggiani,${ }^{71,18}$ E. Polini, ${ }^{28}$ D. Y. T. Pong, ${ }^{106}$ S. Ponrathnam, ${ }^{11}$ P. Popolizio, ${ }^{40}$ E. K. Porter, ${ }^{34}$ R. Poulton, ${ }^{40}$ J. Powell, ${ }^{140}$ M. Pracchia, ${ }^{28}$ T. Pradier, ${ }^{160}$ A. K. Prajapati, ${ }^{77}$ K. Prasai, ${ }^{70}$ R. Prasanna, ${ }^{205}$ G. Pratten, ${ }^{14}$ M. Principe, ${ }^{79,264,94}$ G. A. Prodi, ${ }^{266,89}$ L. Prokhorov, ${ }^{14}$ P. Prosposito, ${ }^{17,118}$ L. Prudenzi, ${ }^{102}$ A. Puecher,${ }^{50,111}$ M. Punturo, ${ }^{72}$ F. Puosi, ${ }^{18,71}$ P. Puppo, ${ }^{48}$ M. Pürrer, ${ }^{102}$ H. Qi,${ }^{17}$ V. Quetschke, ${ }^{148}$ R. Quitzow-James, ${ }^{86}$ F. J. Raab, ${ }^{64}$ G. Raaijmakers, ${ }^{85,50}$ H. Radkins, ${ }^{64}$ N. Radulesco, ${ }^{92}$ P. Raffai, ${ }^{151}$ S. X. Rail, ${ }^{233}$ S. Raja ${ }^{84}$ C. Rajan, ${ }^{84}$ K. E. Ramirez, ${ }^{6}$ T. D. Ramirez, ${ }^{38}$ A. Ramos-Buades, ${ }^{102}$ J. Rana, ${ }^{146}$ P. Rapagnani, ${ }^{95,48}$ U. D. Rapol, ${ }^{267}$ A. Ray, ${ }^{7}$ V. Raymond, ${ }^{17}$ N. Raza, ${ }^{178}$ M. Razzano, ${ }^{71,18}$ J. Read ${ }^{38}$ L. A. Rees, ${ }^{188}$ T. Regimbau ${ }^{28}$ L. Rei, ${ }^{82}$ S. Reid,${ }^{30}$ S. W. Reid, ${ }^{54}$ D. H. Reitze, ${ }^{1,69}$ P. Relton, ${ }^{17}$ A. Renzini, ${ }^{1}$ P. Rettegno, ${ }^{268,22}$ M. Rezac,${ }^{38}$ F. Ricci, ${ }^{95,48}$ D. Richards, ${ }^{139}$ J. W. Richardson, ${ }^{1}$ L. Richardson, ${ }^{183}$ G. Riemenschneider, ${ }^{268,22}$ K. Riles, ${ }^{182}$ S. Rinaldi, ${ }^{18,71}$ K. Rink, ${ }^{178}$ M. Rizzo, ${ }^{15}$ N. A. Robertson, ${ }^{1,66}$ R. Robie, ${ }^{1}$ F. Robinet, ${ }^{39}$ A. Rocchi, ${ }^{118}$ S. Rodriguez,${ }^{38}$ L. Rolland, ${ }^{28}$ J. G. Rollins, ${ }^{1}$ M. Romanelli, ${ }^{96}$ R. Romano, ${ }^{3,4}$ C. L. Romel, ${ }^{64}$ A. Romero-Rodríguez, ${ }^{215}$ I. M. Romero-Shaw, ${ }^{5}$ J. H. Romie, ${ }^{6}$ S. Ronchini, ${ }^{29,98}$ L. Rosa, ${ }^{4,23}$ C. A. Rose, ${ }^{7}$ D. Rosińska, ${ }^{100}$ M. P. Ross, ${ }^{242}$ S. Rowan, ${ }^{66}$ S. J. Rowlinson, ${ }^{14}$ S. Roy, ${ }^{111}$ Santosh Roy, ${ }^{11}$ Soumen Roy, ${ }^{269}$ D. Rozza, ${ }^{115,116}$ P. Ruggi, ${ }^{40}$ K. Ryan, ${ }^{64}$ S. Sachdev, ${ }^{146}$ T. Sadecki, ${ }^{64}$ J. Sadiq, ${ }^{105}$ N. Sago, ${ }^{270}$ S. Saito, ${ }^{21}$ Y. Saito, ${ }^{190}$ K. Sakai, ${ }^{271}$ Y. Sakai, ${ }^{195}$ M. Sakellariadou, ${ }^{51}$ Y. Sakuno, ${ }^{125}$ O. S. Salafia, ${ }^{63,62,61}$ L. Salconi, ${ }^{40}$ M. Saleem, ${ }^{60}$ F. Salemi, ${ }^{88,89}$ A. Samajdar, ${ }^{50,111}$ E. J. Sanchez, ${ }^{1}$ J. H. Sanchez, ${ }^{38}$ L. E. Sanchez, ${ }^{1}$ N. Sanchis-Gual, ${ }^{272}$ J. R. Sanders, ${ }^{273}$ A. Sanuy, ${ }^{27}$ T. R. Saravanan, ${ }^{11}$ N. Sarin, ${ }^{5}$ B. Sassolas, ${ }^{155}$ H. Satari, ${ }^{83}$ S. Sato, ${ }^{274}$ T. Sato, ${ }^{173}$ O. Sauter,${ }^{69}$ R. L. Savage,${ }^{64}$ T. Sawada, ${ }^{202}$ D. Sawant, ${ }^{97}$ H. L. Sawant, ${ }^{11}$ S. Sayah, ${ }^{155}$ D. Schaetzl, ${ }^{1}$ M. Scheel, ${ }^{130}$ J. Scheuer, ${ }^{15}$ M. Schiworski, ${ }^{80}$ P. Schmidt, ${ }^{14}$ S. Schmidt, ${ }^{11}$ R. Schnabel, ${ }^{122}$ M. Schneewind, ${ }^{9,10}$ R. M. S. Schofield, ${ }^{57}$ A. Schönbeck, ${ }^{122}$ B. W. Schulte, ${ }^{9,10}$ B. F. Schutz, ${ }^{17,9,10}$ 
E. Schwartz, ${ }^{17}$ J. Scott, ${ }^{66}$ S. M. Scott, ${ }^{8}$ M. Seglar-Arroyo, ${ }^{28}$ T. Sekiguchi, ${ }^{26}$ Y. Sekiguchi, ${ }^{275}$ D. Sellers, ${ }^{6}$ A. S. Sengupta, ${ }^{269}$ D. Sentenac, ${ }^{40}$ E. G. Seo, ${ }^{106}$ V. Sequino, ${ }^{23,4}$ A. Sergeev, ${ }^{218}$ Y. Setyawati, ${ }^{111}$ T. Shaffer, ${ }^{64}$ M. S. Shahriar, ${ }^{15}$ B. Shams, ${ }^{169}$ L. Shao, ${ }^{199}$ A. Sharma, ${ }^{29,98}$ P. Sharma,${ }^{84}$ P. Shawhan, ${ }^{101}$ N. S. Shcheblanov, ${ }^{237}$ S. Shibagaki, ${ }^{125}$ M. Shikauchi, ${ }^{112}$ R. Shimizu, ${ }^{21}$ T. Shimoda ${ }^{25}$ K. Shimode, ${ }^{190}$ H. Shinkai, ${ }^{276}$ T. Shishido, ${ }^{45}$ A. Shoda, ${ }^{20}$ D. H. Shoemaker,${ }^{67}$ D. M. Shoemaker, ${ }^{165}$ S. ShyamSundar, ${ }^{84}$ M. Sieniawska, ${ }^{100}$ D. Sigg,${ }^{64}$ L. P. Singer, ${ }^{109}$ D. Singh,${ }^{146}$ N. Singh, ${ }^{100}$ A. Singha, ${ }^{152,50}$ A. M. Sintes, ${ }^{142}$ V. Sipala, ${ }^{115,116}$ V. Skliris, ${ }^{17}$ B. J. J. Slagmolen, ${ }^{8}$ T. J. Slaven-Blair, ${ }^{83}$ J. Smetana, ${ }^{14}$ J. R. Smith, ${ }^{38}$ R. J. E. Smith, ${ }^{5}$ J. Soldateschi, ${ }^{277,278,47}$ S. N. Somala, ${ }^{279}$ K. Somiya, ${ }^{216}$ E. J. Son, ${ }^{223}$ K. Soni, ${ }^{11}$ S. Soni, ${ }^{2}$ V. Sordini, ${ }^{134}$ F. Sorrentino, ${ }^{82}$ N. Sorrentino, ${ }^{71,18}$ H. Sotani, ${ }^{280}$ R. Soulard,${ }^{92}$ T. Souradeep, ${ }^{267,11}$ E. Sowell, ${ }^{145}$ V. Spagnuolo, ${ }^{152,50}$ A. P. Spencer, ${ }^{66}$ M. Spera, ${ }^{74,75}$ R. Srinivasan, ${ }^{92}$ A. K. Srivastava, ${ }^{77}$ V. Srivastava, ${ }^{58}$ K. Staats, ${ }^{15}$ C. Stachie, ${ }^{92}$ D. A. Steer, ${ }^{34}$ J. Steinlechner, ${ }^{15,50}$ S. Steinlechner, ${ }^{152,50}$ D. J. Stops, ${ }^{14}$ M. Stover, ${ }^{170}$ K. A. Strain, ${ }^{66}$ L. C. Strang, ${ }^{114}$ G. Stratta, ${ }^{281,47}$ A. Strunk, ${ }^{64}$ R. Sturani, ${ }^{263}$ A. L. Stuver, ${ }^{120}$ S. Sudhagar, ${ }^{11}$ V. Sudhir, ${ }^{67}$ R. Sugimoto, ${ }^{282,204}$ H. G. Suh, ${ }^{7}$ T. Z. Summerscales, ${ }^{283}$ H. Sun, ${ }^{83}$ L. Sun, ${ }^{8}$ S. Sunil, ${ }^{77}$ A. Sur, ${ }^{78}$ J. Suresh, ${ }^{112,35}$ P. J. Sutton, ${ }^{17}$ Takamasa Suzuki, ${ }^{173}$ Toshikazu Suzuki, ${ }^{35}$ B. L. Swinkels, ${ }^{50}$ M. J. Szczepańczyk, ${ }^{69}$ P. Szewczyk, ${ }^{100}$ M. Tacca, ${ }^{50}$ H. Tagoshi, ${ }^{35}$ S. C. Tait, ${ }^{66}$ H. Takahashi, ${ }^{284}$ R. Takahashi, ${ }^{20}$ A. Takamori, ${ }^{37}$ S. Takano, ${ }^{25}$ H. Takeda, ${ }^{25}$ M. Takeda, ${ }^{202}$ C. J. Talbot, ${ }^{30}$ C. Talbot, ${ }^{1}$ H. Tanaka, ${ }^{285}$ Kazuyuki Tanaka, ${ }^{202}$ Kenta Tanaka, ${ }^{285}$ Taiki Tanaka, ${ }^{35}$ Takahiro Tanaka, ${ }^{270}$ A. J. Tanasijczuk, ${ }^{49}$ S. Tanioka, ${ }^{20,45}$ D. B. Tanner, ${ }^{69}$ D. Tao, ${ }^{1}$ L. Tao, ${ }^{69}$ E. N. Tapia San Martín, ${ }^{50,20}$ C. Taranto, ${ }^{117}$ J. D. Tasson, ${ }^{191}$ S. Telada, ${ }^{286}$ R. Tenorio, ${ }^{142}$ J. E. Terhune, ${ }^{120}$ L. Terkowski, ${ }^{122}$ M. P. Thirugnanasambandam, ${ }^{11}$ M. Thomas, ${ }^{6}$ P. Thomas, ${ }^{64}$

J. E. Thompson, ${ }^{17}$ S. R. Thondapu, ${ }^{84}$ K. A. Thorne, ${ }^{6}$ E. Thrane, ${ }^{5}$ Shubhanshu Tiwari, ${ }^{158}$ Srishti Tiwari, ${ }^{11}$ V. Tiwari, ${ }^{17}$ A. M. Toivonen, ${ }^{60}$ K. Toland ${ }^{66}$ A. E. Tolley, ${ }^{153}$ T. Tomaru, ${ }^{20}$ Y. Tomigami,${ }^{202}$ T. Tomura, ${ }^{190}$ M. Tonelli, ${ }^{71,18}$

A. Torres-Forné, ${ }^{121}$ C. I. Torrie, ${ }^{1}$ I. Tosta e Melo, ${ }^{115,116}$ D. Töyrä, ${ }^{8}$ A. Trapananti, ${ }^{247,72}{ }^{4}$. Travasso, ${ }^{72,247}$ G. Traylor, ${ }^{6}$ M. Trevor, ${ }^{101}$ M. C. Tringali, ${ }^{40}$ A. Tripathee, ${ }^{182}$ L. Troiano, ${ }^{287,94}$ A. Trovato,${ }^{34}$ L. Trozzo, ${ }^{4,190}$ R. J. Trudeau, ${ }^{1}$ D. S. Tsai, ${ }^{124}$ D. Tsai, ${ }^{124}$ K. W. Tsang, ${ }^{50,288,111}$ T. Tsang,${ }^{289} \mathrm{~J}-\mathrm{S}$. Tsao, ${ }^{196} \mathrm{M}$. Tse,${ }^{67} \mathrm{R}$. Tso, ${ }^{130} \mathrm{~K}$. Tsubono, ${ }^{25} \mathrm{~S}$. Tsuchida,${ }^{202}$ L. Tsukada, ${ }^{112}$ D. Tsuna, ${ }^{112}$ T. Tsutsui, ${ }^{112}$ T. Tsuzuki, ${ }^{21}$ K. Turbang,,${ }^{290,207}$ M. Turconi, ${ }^{92}$ D. Tuyenbayev, ${ }^{202}$ A. S. Ubhi, ${ }^{14}$ N. Uchikata, ${ }^{35}$ T. Uchiyama, ${ }^{190}$ R. P. Udall, ${ }^{1}$ A. Ueda, ${ }^{185}$ T. Uehara, ${ }^{291,292}$ K. Ueno, ${ }^{112}$ G. Ueshima, ${ }^{293}$ C. S. Unnikrishnan, ${ }^{179}$ F. Uraguchi, ${ }^{21}$ A. L. Urban, ${ }^{2}$ T. Ushiba, ${ }^{190}$ A. Utina,${ }^{152,50}$ H. Vahlbruch, ${ }^{9,10}$ G. Vajente, ${ }^{1}$ A. Vajpeyi, ${ }^{5}$ G. Valdes, ${ }^{183}$ M. Valentini, ${ }^{88,89}$ V. Valsan, ${ }^{7}$ N. van Bakel, ${ }^{50}$ M. van Beuzekom, ${ }^{50}$ J. F. J. van den Brand, ${ }^{152,294,50}$ C. Van Den Broeck, ${ }^{11,50}$

D. C. Vander-Hyde, ${ }^{58}$ L. van der Schaaf,${ }^{50}$ J. V. van Heijningen, ${ }^{49}$ J. Vanosky, ${ }^{1}$ M. H. P. M. van Putten, ${ }^{295}$ N. van Remortel, ${ }^{207}$ M. Vardaro, ${ }^{240,50}$ A. F. Vargas, ${ }^{114}$ V. Varma, ${ }^{177}$ M. Vasúth,${ }^{68}$ A. Vecchio, ${ }^{14}$ G. Vedovato, ${ }^{75}$ J. Veitch,${ }^{66}$ P. J. Veitch, ${ }^{80}$ J. Venneberg, ${ }^{9,10}$ G. Venugopalan, ${ }^{1}$ D. Verkindt, ${ }^{28}$ P. Verma, ${ }^{230}$ Y. Verma,${ }^{84}$ D. Veske, ${ }^{43}$ F. Vetrano, ${ }^{46}$ A. Viceré,${ }^{46,47}$ S. Vidyant ${ }^{58}$ A. D. Viets, ${ }^{246}$ A. Vijaykumar, ${ }^{19}$ V. Villa-Ortega, ${ }^{105}$ J.-Y. Vinet, ${ }^{92}$ A. Virtuoso ${ }^{186,32}$ S. Vitale ${ }^{67}$ T. Vo, ${ }^{58}$ H. Vocca ${ }^{73,72}$ E. R. G. von Reis, ${ }^{64}$ J. S. A. von Wrangel, ${ }^{9,10}$ C. Vorvick, ${ }^{64}$ S. P. Vyatchanin, ${ }^{87}$ L. E. Wade, ${ }^{170}$ M. Wade, ${ }^{170}$ K. J. Wagner, ${ }^{123}$ R. C. Walet,${ }^{50}$ M. Walker,${ }^{54}$ G. S. Wallace, ${ }^{30}$ L. Wallace, ${ }^{1}$ S. Walsh, ${ }^{7}$ J. Wang, ${ }^{174}$ J. Z. Wang, ${ }^{182}$ W. H. Wang ${ }^{148}$ R. L. Ward, ${ }^{8}$ J. Warner ${ }^{64}$ M. Was,${ }^{28}$ T. Washimi,${ }^{20}$ N. Y. Washington, ${ }^{1}$ J. Watchi, ${ }^{143}$ B. Weaver, ${ }^{64}$ S. A. Webster, ${ }^{66}$ M. Weinert, ${ }^{9,10}$ A. J. Weinstein, ${ }^{1}$ R. Weiss,${ }^{67}$ C. M. Weller, ${ }^{242}$ F. Wellmann, ${ }^{9,10}$ L. Wen ${ }^{83}$ P. Weßels, ${ }^{9,10}$ K. Wette, ${ }^{8}$ J. T. Whelan, ${ }^{123}$ D. D. White, ${ }^{38}$ B. F. Whiting, ${ }^{69}$ C. Whittle, ${ }^{67}$ D. Wilken,,${ }^{9,10}$ D. Williams,${ }^{66}$ M. J. Williams,${ }^{66}$ A. R. Williamson, ${ }^{153}$ J. L. Willis, ${ }^{1}$ B. Willke, ${ }^{9,10}$ D. J. Wilson, ${ }^{138}$ W. Winkler, ${ }^{9,10}$ C. C. Wipf, ${ }^{1}$ T. Wlodarczyk, ${ }^{102}$ G. Woan, ${ }^{66}$ J. Woehler, ${ }^{9,10}$ J. K. Wofford, ${ }^{123}$ I. C. F. Wong, ${ }^{106}$ C. Wu, ${ }^{131}$ D. S. Wu, ${ }^{9,10}$ H. Wu, ${ }^{131}$ S. Wu, ${ }^{131}$ D. M. Wysocki, ${ }^{7}$ L. Xiao, ${ }^{1}$ W-R. Xu, ${ }^{196}$ T. Yamada, ${ }^{285}$ H. Yamamoto, ${ }^{1}$ Kazuhiro Yamamoto, ${ }^{189}$ Kohei Yamamoto, ${ }^{285}$ T. Yamamoto, ${ }^{190}$ K. Yamashita, ${ }^{201}$ R. Yamazaki, ${ }^{198}$ F. W. Yang, ${ }^{169}$ L. Yang, ${ }^{163}$ Y. Yang, ${ }^{296}$ Yang Yang, ${ }^{69}$ Z. Yang, ${ }^{60}$ M. J. Yap, ${ }^{8}$ D. W. Yeeles, ${ }^{17}$ A. B. Yelikar, ${ }^{123}$ M. Ying, ${ }^{124}$ K. Yokogawa, ${ }^{201}$ J. Yokoyama, ${ }^{26,25}$ T. Yokozawa, ${ }^{190}$ J. Yoo, ${ }^{177}$ T. Yoshioka, ${ }^{201}$ Hang Yu, ${ }^{130}$ Haocun Yu, ${ }^{67}$ H. Yuzurihara, ${ }^{35}$ A. Zadrożny, ${ }^{230}$ M. Zanolin, ${ }^{33}$ S. Zeidler, ${ }^{297}$ T. Zelenova, ${ }^{40}$ J.-P. Zendri, ${ }^{75}$ M. Zevin, ${ }^{159}$ M. Zhan, ${ }^{174}$ H. Zhang, ${ }^{196}$ J. Zhang, ${ }^{83}$ L. Zhang, ${ }^{1}$ T. Zhang, ${ }^{14}$ Y. Zhang, ${ }^{183}$ C. Zhao, ${ }^{83}$ G. Zhao, ${ }^{143}$ Y. Zhao, ${ }^{20}$ Yue Zhao, ${ }^{169}$ R. Zhou, ${ }^{192}$ Z. Zhou, ${ }^{15}$ X. J. Zhu, ${ }^{5}$ Z.-H. Zhu, ${ }^{113}$ A. B. Zimmerman, ${ }^{165}$ M. E. Zucker, ${ }^{1,67}$ and J. Zweizig ${ }^{1}$

(The LIGO Scientific Collaboration, the Virgo Collaboration, and the KAGRA Collaboration)

\footnotetext{
${ }^{1}$ LIGO Laboratory, California Institute of Technology, Pasadena, California 91125, USA

${ }^{2}$ Louisiana State University, Baton Rouge, Louisiana 70803, USA

${ }^{3}$ Dipartimento di Farmacia, Università di Salerno, I-84084 Fisciano, Salerno, Italy

${ }^{4}$ INFN, Sezione di Napoli, Complesso Universitario di Monte S. Angelo, I-80126 Napoli, Italy
} 
${ }^{5}$ OzGrav, School of Physics and Astronomy, Monash University, Clayton 3800, Victoria, Australia

${ }^{6}$ LIGO Livingston Observatory, Livingston, Louisiana 70754, USA

${ }^{7}$ University of Wisconsin-Milwaukee, Milwaukee, Wisconsin 53201, USA

${ }^{8}$ OzGrav, Australian National University, Canberra, Australian Capital Territory 0200, Australia

${ }^{9}$ Max Planck Institute for Gravitational Physics (Albert Einstein Institute), D-30167 Hannover, Germany

${ }^{10}$ Leibniz Universität Hannover, D-30167 Hannover, Germany

${ }^{11}$ Inter-University Centre for Astronomy and Astrophysics, Pune 411007, India

${ }^{12}$ University of Cambridge, Cambridge CB2 1TN, United Kingdom

${ }^{13}$ Theoretisch-Physikalisches Institut, Friedrich-Schiller-Universität Jena, D-07743 Jena, Germany

${ }^{14}$ University of Birmingham, Birmingham B15 2TT, United Kingdom

${ }^{15}$ Center for Interdisciplinary Exploration and Research in Astrophysics (CIERA), Northwestern University, Evanston, Illinois 60208, USA

${ }^{16}$ Instituto Nacional de Pesquisas Espaciais, 12227-010 São José dos Campos, São Paulo, Brazil

${ }^{17}$ Gravity Exploration Institute, Cardiff University, Cardiff CF24 3AA, United Kingdom

${ }^{18}$ INFN, Sezione di Pisa, I-56127 Pisa, Italy

${ }^{19}$ International Centre for Theoretical Sciences, Tata Institute of Fundamental Research, Bengaluru 560089, India

${ }^{20}$ Gravitational Wave Science Project, National Astronomical Observatory of Japan (NAOJ), Mitaka City, Tokyo 181-8588, Japan

${ }^{21}$ Advanced Technology Center, National Astronomical Observatory of Japan (NAOJ), Mitaka City, Tokyo 181-8588, Japan

${ }^{22}$ INFN Sezione di Torino, I-10125 Torino, Italy

${ }^{23}$ Università di Napoli "Federico II," Complesso Universitario di Monte S. Angelo, I-80126 Napoli, Italy

${ }^{24}$ Université de Lyon, Université Claude Bernard Lyon 1, CNRS, Institut Lumière Matière, F-69622 Villeurbanne, France

${ }^{25}$ Department of Physics, The University of Tokyo, Bunkyo-ku, Tokyo 113-0033, Japan

${ }^{26}$ Research Center for the Early Universe (RESCEU), The University of Tokyo, Bunkyo-ku, Tokyo 113-0033, Japan

${ }^{27}$ Institut de Ciències del Cosmos (ICCUB), Universitat de Barcelona, C/ Martí i Franquès 1, Barcelona 08028, Spain

${ }^{28}$ Laboratoire d'Annecy de Physique des Particules (LAPP), Université Grenoble Alpes, Université Savoie Mont Blanc, CNRS/IN2P3, F-74941 Annecy, France

${ }^{29}$ Gran Sasso Science Institute (GSSI), I-67100 L'Aquila, Italy

${ }^{30}$ SUPA, University of Strathclyde, Glasgow G1 1XQ, United Kingdom

${ }^{31}$ Dipartimento di Scienze Matematiche, Informatiche e Fisiche, Università di Udine, I-33100 Udine, Italy

${ }^{32}$ INFN, Sezione di Trieste, I-34127 Trieste, Italy

${ }^{33}$ Embry-Riddle Aeronautical University, Prescott, Arizona 86301, USA

${ }^{34}$ Université de Paris, CNRS, Astroparticule et Cosmologie, F-75006 Paris, France

${ }^{35}$ Institute for Cosmic Ray Research (ICRR), KAGRA Observatory, The University of Tokyo, Kashiwa City, Chiba 277-8582, Japan

${ }^{36}$ Accelerator Laboratory, High Energy Accelerator Research Organization (KEK), Tsukuba City, Ibaraki 305-0801, Japan

${ }^{37}$ Earthquake Research Institute, The University of Tokyo, Bunkyo-ku, Tokyo 113-0032, Japan

${ }^{38}$ California State University Fullerton, Fullerton, California 92831, USA

${ }^{39}$ Université Paris-Saclay, CNRS/IN2P3, IJCLab, 91405 Orsay, France

${ }^{40}$ European Gravitational Observatory (EGO), I-56021 Cascina, Pisa, Italy

${ }^{41}$ Chennai Mathematical Institute, Chennai 603103, India

${ }^{42}$ Department of Mathematics and Physics, Gravitational Wave Science Project, Hirosaki University, Hirosaki City, Aomori 036-8561, Japan

${ }^{43}$ Columbia University, New York, New York 10027, USA

${ }^{44}$ Kamioka Branch, National Astronomical Observatory of Japan (NAOJ), Kamioka-cho, Hida City, Gifu 506-1205, Japan

${ }^{45}$ The Graduate University for Advanced Studies (SOKENDAI), Mitaka City, Tokyo 181-8588, Japan

${ }^{46}$ Università degli Studi di Urbino "Carlo Bo," I-61029 Urbino, Italy

${ }^{47}$ INFN, Sezione di Firenze, I-50019 Sesto Fiorentino, Firenze, Italy

${ }^{48}$ INFN, Sezione di Roma, I-00185 Roma, Italy

${ }^{49}$ Université catholique de Louvain, B-1348 Louvain-la-Neuve, Belgium

${ }^{50}$ Nikhef, Science Park 105, 1098 XG Amsterdam, Netherlands

${ }^{51}$ King's College London, University of London, London WC2R 2LS, United Kingdom

${ }^{52}$ Korea Institute of Science and Technology Information (KISTI), Yuseong-gu, Daejeon 34141, Korea 


\author{
${ }^{53}$ National Institute for Mathematical Sciences, Yuseong-gu, Daejeon 34047, Korea \\ ${ }^{54}$ Christopher Newport University, Newport News, Virginia 23606, USA \\ ${ }^{55}$ International College, Osaka University, Toyonaka City, Osaka 560-0043, Japan \\ ${ }^{56}$ School of High Energy Accelerator Science, The Graduate University for Advanced Studies \\ (SOKENDAI), Tsukuba City, Ibaraki 305-0801, Japan \\ ${ }^{57}$ University of Oregon, Eugene, Oregon 97403, USA \\ ${ }^{58}$ Syracuse University, Syracuse, New York 13244, USA \\ ${ }^{59}$ Université de Liège, B-4000 Liège, Belgium \\ ${ }^{60}$ University of Minnesota, Minneapolis, Minnesota 55455, USA \\ ${ }^{61}$ Università degli Studi di Milano-Bicocca, I-20126 Milano, Italy \\ ${ }^{62}$ INFN, Sezione di Milano-Bicocca, I-20126 Milano, Italy \\ ${ }^{63}$ INAF, Osservatorio Astronomico di Brera sede di Merate, I-23807 Merate, Lecco, Italy \\ ${ }^{64}$ LIGO Hanford Observatory, Richland, Washington 99352, USA \\ ${ }^{65}$ Dipartimento di Medicina, Chirurgia e Odontoiatria "Scuola Medica Salernitana," \\ Università di Salerno, I-84081 Baronissi, Salerno, Italy \\ ${ }^{66}$ SUPA, University of Glasgow, Glasgow G12 8QQ, United Kingdom \\ ${ }^{67}$ LIGO Laboratory, Massachusetts Institute of Technology, Cambridge, Massachusetts 02139, USA \\ ${ }^{68}$ Wigner RCP, RMKI, H-1121 Budapest, Konkoly Thege Miklós út 29-33, Hungary \\ ${ }^{69}$ University of Florida, Gainesville, Florida 32611, USA \\ ${ }^{70}$ Stanford University, Stanford, California 94305, USA \\ ${ }^{71}$ Università di Pisa, I-56127 Pisa, Italy \\ ${ }^{72}$ INFN, Sezione di Perugia, I-06123 Perugia, Italy \\ ${ }^{73}$ Università di Perugia, I-06123 Perugia, Italy \\ ${ }^{74}$ Università di Padova, Dipartimento di Fisica e Astronomia, I-35131 Padova, Italy \\ ${ }^{75}$ INFN, Sezione di Padova, I-35131 Padova, Italy \\ ${ }^{76}$ Montana State University, Bozeman, Montana 59717, USA \\ ${ }^{77}$ Institute for Plasma Research, Bhat, Gandhinagar 382428, India \\ ${ }^{78}$ Nicolaus Copernicus Astronomical Center, Polish Academy of Sciences, 00-716 Warsaw, Poland \\ ${ }^{79}$ Dipartimento di Ingegneria, Università del Sannio, I-82100 Benevento, Italy \\ ${ }^{80}$ OzGrav, University of Adelaide, Adelaide, South Australia 5005, Australia \\ ${ }^{81}$ California State University, Los Angeles, 5151 State University Drive, Los Angeles, California 90032, USA \\ ${ }^{82}$ INFN, Sezione di Genova, I-16146 Genova, Italy \\ ${ }^{83} \mathrm{OzGrav}$, University of Western Australia, Crawley, Western Australia 6009, Australia \\ ${ }^{84}$ RRCAT, Indore, Madhya Pradesh 452013, India \\ ${ }^{85}$ GRAPPA, Anton Pannekoek Institute for Astronomy and Institute for High-Energy Physics, \\ University of Amsterdam, Science Park 904, 1098 XH Amsterdam, Netherlands \\ ${ }^{86}$ Missouri University of Science and Technology, Rolla, Missouri 65409, USA \\ ${ }^{87}$ Faculty of Physics, Lomonosov Moscow State University, Moscow 119991, Russia \\ ${ }^{88}$ Dipartimento di Fisica, Università di Trento, I-38123 Povo, Trento, Italy \\ ${ }^{89}$ INFN, Trento Institute for Fundamental Physics and Applications, I-38123 Povo, Trento, Italy \\ ${ }^{90}$ SUPA, University of the West of Scotland, Paisley PA1 2BE, United Kingdom \\ ${ }^{91}$ Bar-Ilan University, Ramat Gan 5290002, Israel \\ ${ }^{92}$ Artemis, Université Côte d'Azur, Observatoire de la Côte d'Azur, CNRS, F-06304 Nice, France \\ ${ }^{93}$ Dipartimento di Fisica “E.R. Caianiello," Università di Salerno, I-84084 Fisciano, Salerno, Italy \\ ${ }^{94}$ INFN, Sezione di Napoli, Gruppo Collegato di Salerno, Complesso Universitario di Monte S. Angelo, \\ I-80126 Napoli, Italy \\ ${ }^{95}$ Università di Roma “La Sapienza," I-00185 Roma, Italy \\ ${ }^{96}$ Université Rennes, CNRS, Institut FOTON-UMR6082, F-3500 Rennes, France \\ ${ }^{97}$ Indian Institute of Technology Bombay, Powai, Mumbai 400 076, India \\ ${ }^{98}$ Laboratori Nazionali del Gran Sasso, INFN, I-67100 Assergi, Italy \\ ${ }^{99}$ Laboratoire Kastler Brossel, Sorbonne Université, CNRS, ENS-Université PSL, Collège de France, \\ F-75005 Paris, France \\ ${ }^{100}$ Astronomical Observatory Warsaw University, 00-478 Warsaw, Poland \\ ${ }^{101}$ University of Maryland, College Park, Maryland 20742, USA \\ ${ }^{102}$ Max Planck Institute for Gravitational Physics (Albert Einstein Institute), D-14476 Potsdam, Germany \\ ${ }^{103}$ L2IT, Laboratoire des 2 Infinis-Toulouse, Université de Toulouse, \\ CNRS/IN2P3, UPS, F-31062 Toulouse Cedex 9, France \\ ${ }^{104}$ School of Physics, Georgia Institute of Technology, Atlanta, Georgia 30332, USA \\ ${ }^{105}$ IGFAE, Campus Sur, Universidade de Santiago de Compostela, 15782 Santiago, Spain \\ ${ }^{106}$ The Chinese University of Hong Kong, Shatin, NT, Hong Kong
}




\author{
${ }^{107}$ Stony Brook University, Stony Brook, New York 11794, USA \\ ${ }^{108}$ Center for Computational Astrophysics, Flatiron Institute, New York, New York 10010, USA \\ ${ }^{109}$ NASA Goddard Space Flight Center, Greenbelt, Maryland 20771, USA \\ ${ }^{110}$ Dipartimento di Fisica, Università degli Studi di Genova, I-16146 Genova, Italy \\ ${ }^{111}$ Institute for Gravitational and Subatomic Physics (GRASP), Utrecht University, \\ Princetonplein 1, 3584 CC Utrecht, Netherlands \\ ${ }^{112}$ RESCEU, University of Tokyo, Tokyo 113-0033, Japan \\ ${ }^{113}$ Department of Astronomy, Beijing Normal University, Beijing 100875, China \\ ${ }^{114} \mathrm{OzGrav}$, University of Melbourne, Parkville, Victoria 3010, Australia \\ ${ }^{115}$ Università degli Studi di Sassari, I-07100 Sassari, Italy \\ ${ }^{116}$ Laboratori Nazionali del Sud, INFN, I-95125 Catania, Italy \\ ${ }^{117}$ Università di Roma Tor Vergata, I-00133 Roma, Italy \\ ${ }^{118}$ INFN, Sezione di Roma Tor Vergata, I-00133 Roma, Italy \\ ${ }^{119}$ University of Sannio at Benevento, I-82100 Benevento, Italy \\ and INFN, Sezione di Napoli, I-80100 Napoli, Italy \\ ${ }^{120}$ Villanova University, 800 Lancaster Avenue, Villanova, Pennsylvania 19085, USA \\ ${ }^{121}$ Departamento de Astronomía y Astrofísica, Universitat de València, \\ E-46100 Burjassot, València, Spain \\ ${ }^{122}$ Universität Hamburg, D-22761 Hamburg, Germany \\ ${ }^{123}$ Rochester Institute of Technology, Rochester, New York 14623, USA \\ ${ }^{124}$ National Tsing Hua University, Hsinchu City, 30013 Taiwan, Republic of China \\ ${ }^{125}$ Department of Applied Physics, Fukuoka University, Jonan, Fukuoka City, Fukuoka 814-0180, Japan \\ ${ }^{126}$ OzGrav, Charles Sturt University, Wagga Wagga, New South Wales 2678, Australia \\ ${ }^{127}$ Department of Physics, Tamkang University, Danshui District, New Taipei City 25137, Taiwan \\ ${ }^{128}$ Department of Physics and Institute of Astronomy, National Tsing Hua University, \\ Hsinchu 30013, Taiwan \\ ${ }^{129}$ Department of Physics, Center for High Energy and High Field Physics, National Central University, \\ Zhongli District, Taoyuan City 32001, Taiwan \\ ${ }^{130}$ CaRT, California Institute of Technology, Pasadena, California 91125, USA \\ ${ }^{131}$ Department of Physics, National Tsing Hua University, Hsinchu 30013, Taiwan \\ ${ }^{132}$ Dipartimento di Ingegneria Industriale (DIIN), Università di Salerno, I-84084 Fisciano, Salerno, Italy \\ ${ }^{133}$ Institute of Physics, Academia Sinica, Nankang, Taipei 11529, Taiwan \\ ${ }^{134}$ Université Lyon, Université Claude Bernard Lyon 1, CNRS, IP2I Lyon/IN2P3, UMR 5822, \\ F-69622 Villeurbanne, France \\ ${ }^{135}$ Seoul National University, Seoul 08826, South Korea \\ ${ }^{136}$ Pusan National University, Busan 46241, South Korea \\ ${ }^{137}$ Osservatorio Astronomico di Padova, INAF, I-35122 Padova, Italy \\ ${ }^{138}$ University of Arizona, Tucson, Arizona 85721, USA \\ ${ }^{139}$ Rutherford Appleton Laboratory, Didcot OX11 ODE, United Kingdom \\ ${ }^{140}$ OzGrav, Swinburne University of Technology, Hawthorn VIC 3122, Australia \\ ${ }^{141}$ Université libre de Bruxelles, Avenue Franklin Roosevelt 50-1050 Bruxelles, Belgium \\ ${ }^{142}$ Universitat de les Illes Balears, IAC3-IEEC, E-07122 Palma de Mallorca, Spain \\ ${ }^{143}$ Université Libre de Bruxelles, Brussels 1050, Belgium \\ ${ }^{144}$ Departamento de Matemáticas, Universitat de València, E-46100 Burjassot, València, Spain \\ ${ }^{145}$ Texas Tech University, Lubbock, Texas 79409, USA \\ ${ }^{146}$ The Pennsylvania State University, University Park, Pennsylvania 16802, USA \\ ${ }^{147}$ University of Rhode Island, Kingston, Rhode Island 02881, USA \\ ${ }^{148}$ The University of Texas Rio Grande Valley, Brownsville, Texas 78520, USA \\ ${ }^{149}$ Bellevue College, Bellevue, Washington 98007, USA \\ ${ }^{150}$ Scuola Normale Superiore, Piazza dei Cavalieri, 7-56126 Pisa, Italy \\ ${ }^{151}$ MTA-ELTE Astrophysics Research Group, Institute of Physics, Eötvös University, \\ Budapest 1117, Hungary \\ ${ }^{152}$ Maastricht University, P.O. Box 616, 6200 MD Maastricht, Netherlands \\ ${ }^{153}$ University of Portsmouth, Portsmouth, PO1 3FX, United Kingdom \\ ${ }^{154}$ The University of Sheffield, Sheffield S10 2TN, United Kingdom \\ ${ }^{155}$ Université Lyon, Université Claude Bernard Lyon 1, CNRS, Laboratoire des Matériaux Avancés (LMA), \\ IP2I Lyon/IN2P3, UMR 5822, F-69622 Villeurbanne, France \\ ${ }^{156}$ Dipartimento di Scienze Matematiche, Fisiche e Informatiche, Università di Parma, \\ I-43124 Parma, Italy \\ ${ }^{157}$ INFN, Sezione di Milano Bicocca, Gruppo Collegato di Parma, I-43124 Parma, Italy
}


${ }^{158}$ Physik-Institut, University of Zurich, Winterthurerstrasse 190, 8057 Zurich, Switzerland

${ }^{159}$ University of Chicago, Chicago, Illinois 60637, USA

${ }^{160}$ Université de Strasbourg, CNRS, IPHC UMR 7178, F-67000 Strasbourg, France

${ }^{161}$ West Virginia University, Morgantown, West Virginia 26506, USA

${ }^{162}$ Montclair State University, Montclair, New Jersey 07043, USA

${ }^{163}$ Colorado State University, Fort Collins, Colorado 80523, USA

${ }^{164}$ Institute for Nuclear Research, Hungarian Academy of Sciences, Bem t'er 18/c, H-4026 Debrecen, Hungary

${ }^{165}$ Department of Physics, University of Texas, Austin, Texas 78712, USA

${ }^{166}$ CNR-SPIN, clo Università di Salerno, I-84084 Fisciano, Salerno, Italy

${ }^{167}$ Scuola di Ingegneria, Università della Basilicata, I-85100 Potenza, Italy

${ }^{168}$ Observatori Astronòmic, Universitat de València, E-46980 Paterna, València, Spain

${ }^{169}$ The University of Utah, Salt Lake City, Utah 84112, USA

${ }^{170}$ Kenyon College, Gambier, Ohio 43022, USA

${ }^{171}$ Vrije Universiteit Amsterdam, 1081 HV Amsterdam, Netherlands

${ }^{172}$ Department of Astronomy, The University of Tokyo, Mitaka City, Tokyo 181-8588, Japan

${ }^{173}$ Faculty of Engineering, Niigata University, Nishi-ku, Niigata City, Niigata 950-2181, Japan

${ }^{174}$ State Key Laboratory of Magnetic Resonance and Atomic and Molecular Physics, Innovation Academy

for Precision Measurement Science and Technology (APM), Chinese Academy of Sciences, Xiao Hong Shan, Wuhan 430071, China

${ }^{175}$ University of Szeged, Dóm tér 9, Szeged 6720, Hungary

${ }^{176}$ Universiteit Gent, B-9000 Gent, Belgium

${ }^{177}$ Cornell University, Ithaca, New York 14850, USA

${ }^{178}$ University of British Columbia, Vancouver, British Columbia V6T 1Z4, Canada

${ }^{179}$ Tata Institute of Fundamental Research, Mumbai 400005, India

${ }^{180}$ Osservatorio Astronomico di Capodimonte, INAF, I-80131 Napoli, Italy

${ }^{181}$ The University of Mississippi, University, Mississippi 38677, USA

${ }^{182}$ University of Michigan, Ann Arbor, Michigan 48109, USA

${ }^{183}$ Texas A\&M University, College Station, Texas 77843, USA

${ }^{184}$ Department of Physics, Ulsan National Institute of Science and Technology (UNIST), Ulju-gun, Ulsan 44919, Korea

${ }^{185}$ Applied Research Laboratory, High Energy Accelerator Research Organization (KEK), Tsukuba City, Ibaraki 305-0801, Japan

${ }^{186}$ Dipartimento di Fisica, Università di Trieste, I-34127 Trieste, Italy

${ }^{187}$ Shanghai Astronomical Observatory, Chinese Academy of Sciences, Shanghai 200030, China

${ }^{188}$ American University, Washington, D.C. 20016, USA

${ }^{189}$ Faculty of Science, University of Toyama, Toyama City, Toyama 930-8555, Japan

${ }^{190}$ Institute for Cosmic Ray Research (ICRR), KAGRA Observatory, The University of Tokyo, Kamioka-cho, Hida City, Gifu 506-1205, Japan

${ }^{191}$ Carleton College, Northfield, Minnesota 55057, USA

${ }^{192}$ University of California, Berkeley, California 94720, USA

${ }^{193}$ Maastricht University, 6200 MD Maastricht, Netherlands

${ }^{194}$ College of Industrial Technology, Nihon University, Narashino City, Chiba 275-8575, Japan

${ }^{195}$ Graduate School of Science and Technology, Niigata University, Nishi-ku, Niigata City, Niigata 950-2181, Japan

${ }^{196}$ Department of Physics, National Taiwan Normal University, section IV, Taipei 116, Taiwan

${ }^{197}$ Astronomy and Space Science, Chungnam National University, Yuseong-gu, Daejeon 34134, Korea, Korea

${ }^{198}$ Department of Physics and Mathematics, Aoyama Gakuin University, Sagamihara City, Kanagawa 252-5258, Japan

${ }^{199}$ Kavli Institute for Astronomy and Astrophysics, Peking University, Haidian District, Beijing 100871, China

${ }^{200}$ Yukawa Institute for Theoretical Physics (YITP), Kyoto University, Sakyou-ku, Kyoto City, Kyoto 606-8502, Japan

${ }^{201}$ Graduate School of Science and Engineering, University of Toyama, Toyama City, Toyama 930-8555, Japan

${ }^{202}$ Department of Physics, Graduate School of Science, Osaka City University, Sumiyoshi-ku, Osaka City, Osaka 558-8585, Japan

${ }^{203}$ Nambu Yoichiro Institute of Theoretical and Experimental Physics (NITEP), Osaka City University, Sumiyoshi-ku, Osaka City, Osaka 558-8585, Japan 


\author{
${ }^{204}$ Institute of Space and Astronautical Science (JAXA), \\ Chuo-ku, Sagamihara City, Kanagawa 252-0222, Japan \\ ${ }^{205}$ Directorate of Construction, Services and Estate Management, Mumbai 400094, India \\ ${ }^{206}$ Vanderbilt University, Nashville, Tennessee 37235, USA \\ ${ }^{207}$ Universiteit Antwerpen, Prinsstraat 13, 2000 Antwerpen, Belgium \\ ${ }^{208}$ University of Biatystok, 15-424 Biatystok, Poland \\ ${ }^{209}$ Department of Physics, Ewha Womans University, Seodaemun-gu, Seoul 03760, Korea \\ ${ }^{210}$ National Astronomical Observatories, Chinese Academic of Sciences, \\ Chaoyang District, Beijing 100012, China \\ ${ }^{211}$ School of Astronomy and Space Science, University of Chinese Academy of Sciences, \\ Chaoyang District, Beijing 100012, China \\ ${ }^{212}$ University of Southampton, Southampton SO17 1BJ, United Kingdom \\ ${ }^{213}$ Institute for Cosmic Ray Research (ICRR), The University of Tokyo, \\ Kashiwa City, Chiba 277-8582, Japan \\ ${ }^{214}$ Chung-Ang University, Seoul 06974, South Korea \\ ${ }^{215}$ Institut de Física d'Altes Energies (IFAE), Barcelona Institute of Science and Technology, \\ and ICREA, E-08193 Barcelona, Spain \\ ${ }^{216}$ Graduate School of Science, Tokyo Institute of Technology, Meguro-ku, Tokyo 152-8551, Japan \\ ${ }^{217}$ University of Washington Bothell, Bothell, Washington 98011, USA \\ ${ }^{218}$ Institute of Applied Physics, Nizhny Novgorod 603950, Russia \\ ${ }^{219}$ Ewha Womans University, Seoul 03760, South Korea \\ ${ }^{220}$ Inje University Gimhae, South Gyeongsang 50834, South Korea \\ ${ }^{221}$ Department of Physics, Myongji University, Yongin 17058, Korea \\ ${ }^{222}$ Korea Astronomy and Space Science Institute, Daejeon 34055, South Korea \\ ${ }^{223}$ National Institute for Mathematical Sciences, Daejeon 34047, South Korea \\ ${ }^{224}$ Ulsan National Institute of Science and Technology, Ulsan 44919, South Korea \\ ${ }^{225}$ Department of Physical Science, Hiroshima University, \\ Higashihiroshima City, Hiroshima 903-0213, Japan \\ ${ }^{226}$ School of Physics and Astronomy, Cardiff University, Cardiff, CF24 3AA, United Kingdom \\ ${ }^{227}$ Institute of Astronomy, National Tsing Hua University, Hsinchu 30013, Taiwan \\ ${ }^{228}$ Bard College, 30 Campus Road, Annandale-On-Hudson, New York 12504, USA \\ ${ }^{229}$ Institute of Mathematics, Polish Academy of Sciences, 00656 Warsaw, Poland \\ ${ }^{230}$ National Center for Nuclear Research, 05-400 Świerk-Otwock, Poland \\ ${ }^{231}$ Instituto de Fisica Teorica, 28049 Madrid, Spain \\ ${ }^{232}$ Department of Physics, Nagoya University, Chikusa-ku, Nagoya, Aichi 464-8602, Japan \\ ${ }^{233}$ Université de Montréal/Polytechnique, Montreal, Quebec H3T 1J4, Canada \\ ${ }^{234}$ Laboratoire Lagrange, Université Côte d'Azur, Observatoire Côte d'Azur, CNRS, \\ F-06304 Nice, France \\ ${ }^{235}$ Department of Physics, Hanyang University, Seoul 04763, Korea \\ ${ }^{236}$ Sungkyunkwan University, Seoul 03063, South Korea \\ ${ }^{237}$ NAVIER, École des Ponts, Université Gustave Eiffel, CNRS, 77454 Marne-la-Vallée, France \\ ${ }^{238}$ Department of Physics, National Cheng Kung University, Tainan City 701, Taiwan \\ ${ }^{239}$ National Center for High-performance computing, National Applied Research Laboratories, Hsinchu \\ Science Park, Hsinchu City 30076, Taiwan \\ ${ }^{240}$ Institute for High-Energy Physics, University of Amsterdam, \\ Science Park 904, 1098 XH Amsterdam, Netherlands \\ ${ }^{241}$ NASA Marshall Space Flight Center, Huntsville, Alabama 35811, USA \\ ${ }^{242}$ University of Washington, Seattle, Washington 98195, USA \\ ${ }^{243}$ Dipartimento di Matematica e Fisica, Università degli Studi Roma Tre, I-00146 Roma, Italy \\ ${ }^{244}$ INFN, Sezione di Roma Tre, I-00146 Roma, Italy \\ ${ }^{245}$ ESPCI, CNRS, F-75005 Paris, France \\ ${ }^{246}$ Concordia University Wisconsin, Mequon, Wisconsin 53097, USA \\ ${ }^{247}$ Università di Camerino, Dipartimento di Fisica, I-62032 Camerino, Italy \\ ${ }^{248}$ School of Physics Science and Engineering, Tongji University, Shanghai 200092, China \\ ${ }^{249}$ Southern University and A\&M College, Baton Rouge, Louisiana 70813, USA \\ ${ }^{250}$ Centre Scientifique de Monaco, 8 quai Antoine Ier, MC-98000, Monaco \\ ${ }^{251}$ Institute for Photon Science and Technology, The University of Tokyo, \\ Bunkyo-ku, Tokyo 113-8656, Japan \\ ${ }^{252}$ Indian Institute of Technology Madras, Chennai 600036, India \\ ${ }^{253}$ Saha Institute of Nuclear Physics, Bidhannagar, West Bengal 700064, India
}


${ }^{254}$ The Applied Electromagnetic Research Institute, National Institute of Information and Communications Technology (NICT), Koganei City, Tokyo 184-8795, Japan

${ }^{255}$ Institut des Hautes Etudes Scientifiques, F-91440 Bures-sur-Yvette, France

${ }^{256}$ Faculty of Law, Ryukoku University, Fushimi-ku, Kyoto City, Kyoto 612-8577, Japan

${ }^{257}$ Indian Institute of Science Education and Research, Kolkata, Mohanpur, West Bengal 741252, India

${ }^{258}$ Department of Astrophysics/IMAPP, Radboud University Nijmegen, P.O. Box 9010, 6500 GL Nijmegen, Netherlands

${ }^{259}$ Department of Physics, University of Notre Dame, Notre Dame, Indiana 46556, USA

${ }^{260}$ Consiglio Nazionale delle Ricerche-Istituto dei Sistemi Complessi, Piazzale Aldo Moro 5, I-00185 Roma, Italy

${ }^{261}$ Korea Astronomy and Space Science Institute (KASI), Yuseong-gu, Daejeon 34055, Korea

${ }^{262}$ Hobart and William Smith Colleges, Geneva, New York 14456, USA

${ }^{263}$ International Institute of Physics, Universidade Federal do Rio Grande do Norte, Natal RN 59078-970, Brazil

${ }^{264}$ Museo Storico della Fisica e Centro Studi e Ricerche "Enrico Fermi," I-00184 Roma, Italy

${ }^{265}$ Lancaster University, Lancaster LA1 4YW, United Kingdom

${ }^{266}$ Università di Trento, Dipartimento di Matematica, I-38123 Povo, Trento, Italy

${ }^{267}$ Indian Institute of Science Education and Research, Pune, Maharashtra 411008, India

${ }^{268}$ Dipartimento di Fisica, Università degli Studi di Torino, I-10125 Torino, Italy

${ }^{269}$ Indian Institute of Technology, Palaj, Gandhinagar, Gujarat 382355, India

${ }^{270}$ Department of Physics, Kyoto University, Sakyou-ku, Kyoto City, Kyoto 606-8502, Japan

${ }^{271}$ Department of Electronic Control Engineering, National Institute of Technology, Nagaoka College, Nagaoka City, Niigata 940-8532, Japan

${ }^{272}$ Departamento de Matemática da Universidade de Aveiro and Centre for Research and Development in Mathematics and Applications, Campus de Santiago, 3810-183 Aveiro, Portugal

${ }^{273}$ Marquette University, 11420 W. Clybourn Street, Milwaukee, Wisconsin 53233, USA

${ }^{274}$ Graduate School of Science and Engineering, Hosei University, Koganei City, Tokyo 184-8584, Japan

${ }^{275}$ Faculty of Science, Toho University, Funabashi City, Chiba 274-8510, Japan

${ }^{276}$ Faculty of Information Science and Technology, Osaka Institute of Technology, Hirakata City, Osaka 573-0196, Japan

${ }^{277}$ Università di Firenze, Sesto Fiorentino I-50019, Italy

${ }^{278}$ INAF, Osservatorio Astrofisico di Arcetri, Largo E. Fermi 5, I-50125 Firenze, Italy

${ }^{279}$ Indian Institute of Technology Hyderabad, Sangareddy, Khandi, Telangana 502285, India

${ }^{280}$ iTHEMS (Interdisciplinary Theoretical and Mathematical Sciences Program), The Institute of Physical and Chemical Research (RIKEN), Wako, Saitama 351-0198, Japan

${ }^{281}$ Osservatorio di Astrofisica e Scienza dello Spazio, INAF, I-40129 Bologna, Italy

${ }^{282}$ Department of Space and Astronautical Science, The Graduate University for Advanced Studies (SOKENDAI), Sagamihara City, Kanagawa 252-5210, Japan

${ }^{283}$ Andrews University, Berrien Springs, Michigan 49104, USA

${ }^{284}$ Research Center for Space Science, Advanced Research Laboratories, Tokyo City University, Setagaya, Tokyo 158-0082, Japan

${ }^{285}$ Institute for Cosmic Ray Research (ICRR), Research Center for Cosmic Neutrinos (RCCN), The University of Tokyo, Kashiwa City, Chiba 277-8582, Japan

${ }^{286}$ National Metrology Institute of Japan, National Institute of Advanced Industrial Science and Technology, Tsukuba City, Ibaraki 305-8568, Japan

${ }^{287}$ Dipartimento di Scienze Aziendali-Management and Innovation Systems (DISA-MIS), Università di Salerno, I-84084 Fisciano, Salerno, Italy

${ }^{288}$ Van Swinderen Institute for Particle Physics and Gravity, University of Groningen, Nijenborgh 4, 9747 AG Groningen, Netherlands

${ }^{289}$ Faculty of Science, Department of Physics, The Chinese University of Hong Kong, Shatin, NT, Hong Kong

${ }^{290}$ Vrije Universiteit Brussel, Boulevard de la Plaine 2, 1050 Ixelles, Belgium

${ }^{291}$ Department of Communications Engineering, National Defense Academy of Japan, Yokosuka City, Kanagawa 239-8686, Japan

${ }^{292}$ Department of Physics, University of Florida, Gainesville, Florida 32611, USA

${ }^{293}$ Department of Information and Management Systems Engineering, Nagaoka University of Technology, Nagaoka City, Niigata 940-2188, Japan

${ }^{294}$ Vrije Universiteit Amsterdam, 1081 HV Amsterdam, Netherlands 
${ }^{295}$ Department of Physics and Astronomy, Sejong University, Gwangjin-gu, Seoul 143-747, Korea

${ }^{296}$ Department of Electrophysics, National Chiao Tung University, Hsinchu 300, Taiwan

${ }^{297}$ Department of Physics, Rikkyo University, Toshima-ku, Tokyo 171-8501, Japan

${ }^{\mathrm{a}}$ Deceased, August 2020. 